Pure and Applied Mathematics Quarterly

Volume 6, Number 2

(Special Issue: In honor of

Michael Atiyah and Isadore Singer)

$501-544,2010$

\title{
A Compactly Supported Formula for Equivariant Localization and Simplicial Complexes of Białynicki-Birula Decompositions
}

\author{
Allen Knutson \\ Dedicated to Sir Michael Atiyah and to Isadore Singer on their 80th and 85th \\ birthdays
}

\begin{abstract}
The Duistermaat-Heckman formula for their induced measure on a moment polytope is nowadays seen as the Fourier transform of the AtiyahBott/Berline-Vergne localization formula, applied to the T-equivariant Liouville class. From this formula one does not see directly that the measure is positive, and vanishes outside the moment polytope.

In [Knutson99] we gave a formula for the Duistermaat-Heckman measure whose terms are all positive and compactly supported, using a Morse decomposition. Its derivation required that the stable and unstable Morse strata intersect transversely.

In this paper, we remove this very restrictive condition, at the cost of working with an "iterated" Morse (or Białynicki-Birula) decomposition. This leads in a natural way to a simplicial complex of "closure chains", which in the toric variety case is just a pulling triangulation of the moment polytope. To handle the singularities of the closed strata we restrict to the projective algebraic setting. Conversely, this allows us to work from the beginning with singular projective schemes over algebraically closed ground fields.
\end{abstract}

Received January 28, 2008.

Supported by an NSF grant. 


\section{Contents}

1. Background, and statement of results 502

1.1. The basic formula $\quad 505$

1.2. Examples of $\Delta(X, S) \quad 507$

$\begin{array}{lll}\text { 1.3. The coefficients } v_{\gamma} & 515\end{array}$

1.4. Integrating more general classes $\quad 516$

2. Background on D-H measures and B-B decompositions 518

2.1. D-H measures and equivariant Chow theory 518

2.2. B-B decompositions 521

3. Proofs of the main theorems 525

3.1. Supporting fixed points and closure chains in the B-B decomposition $\quad 525$

3.2. The main theorems 531

4. Constraints on the coefficients $v_{\gamma} \quad 537$

4.1. An easy case of the multiplicities $v(Z)_{\left(f_{0}, f_{1}\right), Y} \quad 537$

4.2. Linear relations among the $\left\{v_{\gamma}\right\} \quad 538$

4.3. Assembling the coefficients $\left\{v_{\bar{\gamma}}\right\} \quad 541$

$\begin{array}{lr}\text { Acknowledgements } & 542\end{array}$

$\begin{array}{ll}\text { References } & 542\end{array}$

\section{BACKGROUnd, AND STATEMENT OF RESUlts}

Let $\mathrm{X} \subseteq \mathbb{P} V$ be a projective algebraic variety over an algebraically closed field, invariant under the linear action of a torus $\mathrm{T}$ on $\mathrm{V}$. Then (as in [BP90]) there is an associated Duistermaat-Heckman measure $\mathrm{DH}(\mathrm{X}, \mathrm{T})$ on the dual $\mathfrak{t}^{*}$ of the Lie algebra, the weak limit as $n \rightarrow \infty$ of the Dirac measures

$$
\sum_{\mu \in T^{*} \subseteq \mathfrak{t}^{*}} \frac{\operatorname{dim}(\mu \text {-weight space in } \Gamma(X ; \mathcal{O}(n)))}{n^{\operatorname{dim} X-\operatorname{dim} T}} \delta_{\mu / n} .
$$


As shown in [BP90], this measure $\mathrm{DH}(\mathrm{X}, \mathrm{T})$ is supported on the convex hull of the weights of $\mathrm{T}$ acting on the lines $\left.\mathcal{O}(1)\right|_{x \in \mathrm{X}^{\top}}$ over the fixed points, and is a piecewise-polynomial times Lebesgue measure on that polytope, called the moment polytope. It is a pleasant way to encode the asymptotics of the Trepresentation $\Gamma(X ; \mathcal{O}(n)), n \rightarrow \infty$.

In the simplest case, $T=1$, this gives a Dirac measure times the leading coefficient $\operatorname{deg} X /(\operatorname{dim} X)$ ! of the Hilbert polynomial. More generally, the value of this function at a interior integral point $p$ of this polytope is the leading coefficient of the Hilbert polynomial of the geometric invariant theory quotient $X / / p T$, with the linearization on $\mathcal{O}(1)$ twisted by the character $-p{ }^{1}$ (One can also extend this definition to rational $p$, and we will state a more general result of this type in proposition 1.2.)

If $\mathrm{T}^{\prime} \rightarrow \mathrm{T}$ is a homomorphism (e.g. the inclusion of a subtorus), then there is a natural map $\mathfrak{t}^{*} \rightarrow \mathfrak{t}^{\prime *}$ taking $\mathrm{DH}(X, T)$ to $\mathrm{DH}\left(X, \mathrm{~T}^{\prime}\right)$. For example, the $\mathrm{T}^{\prime}=1$ case lets one compute the degree using the total mass of the Duistermaat-Heckman measure.

The polytope and measure are named for their origins in the case that the base field is $\mathbb{C}$ [DH82]. If one chooses a Hermitian metric on $\mathrm{V}$ invariant under the compact subgroup $\mathrm{T}_{\mathbb{R}}$ of $\mathrm{T}$, then there is a moment map $\Phi_{\mathrm{T}}: \mathrm{X} \rightarrow \mathfrak{t}^{*}$ whose image is exactly the moment polytope, and $\mathrm{DH}(\mathrm{X}, \mathrm{T})$ is the pushforward along $\Phi_{\mathrm{T}}$ of the Liouville measure on the (smooth part of the) variety $X$. One property of this map $\Phi_{\mathrm{T}}$ is that for $f \in X^{\top}$, the value $\Phi_{\mathrm{T}}(f) \in \mathrm{T}^{*}$ is the $\mathrm{T}$-weight on the line $\left.\mathcal{O}(1)\right|_{\mathrm{f}}$; as such we will use $\Phi_{\mathrm{T}}(\mathrm{f})$ to denote this weight even when the base field is not $\mathbb{C}$ (though $\Phi_{\mathrm{T}}(x)$ will not be defined for $x \notin X^{\top}$ ).

Hereafter we assume the fixed point set $\mathrm{X}^{\top}$ is isolated. Under this assumption Duistermaat and Heckman gave a formula for their measure as an alternating sum over $\mathrm{X}^{\top}$ (this version is from [GLS88, proposition 3.3 and its preceding theorem]):

Theorem. [DH82, GLS88] Let X be a compact symplectic manifold of dimension $2 \mathrm{n}$ with symplectic form $\mathrm{\omega}$ and Liouville measure $\left[\mathrm{\omega}^{\mathrm{n}}\right]$, and $\mathrm{T}$-moment map $\Phi_{\mathrm{T}}$ : $X \rightarrow \mathfrak{t}^{*}$.

\footnotetext{
${ }^{1}$ The quotient $\mathrm{X} / / \mathrm{p} \mathrm{T}$ may only carry a 1-dimensional sheaf, rather than a line bundle, but this does not affect the definitions in any appreciable way.
} 
For each fixed point $\mathrm{f} \in \mathrm{X}^{\mathrm{\top}}$, let $\lambda_{1}^{\mathrm{f}}, \ldots, \lambda_{\mathfrak{n}}^{\mathrm{f}}$ be the weights of $\mathrm{T}$ acting on the tangent space $\mathrm{T}_{\mathrm{f}} \mathrm{X}$. Pick $\vec{v} \in \mathfrak{t}$ such that $\left\langle\vec{v}, \lambda_{\mathfrak{i}}^{f}\right\rangle \neq 0$ for all $\mathrm{f} \in \mathrm{X}^{\mathrm{\top}}, \mathfrak{i}=1, \ldots \mathrm{n}$. (In particular each $\lambda_{i}^{f} \neq 0$, which is the condition that $\mathrm{X}^{\top}$ is isolated.)

Then the measure $\mathrm{DH}(\mathrm{X}, \mathrm{T}):=\left(\Phi_{\mathrm{T}}\right)_{*}\left(\frac{\left[\omega^{\mathfrak{n}}\right]}{\mathrm{n} !}\right)$ on the moment polytope $\Phi_{\mathrm{T}}(\mathrm{X})$ equals the sum

$$
\sum_{f \in X^{\top}} \operatorname{sign}\left(\prod_{i=1}^{n}\left\langle\vec{v}, \lambda_{f}^{i}\right\rangle\right)\left(C_{f}\right)_{*}\left(\text { Lebesgue measure on the orthant } \mathbb{R}_{\geq 0}^{n}\right)
$$

where $\mathrm{C}_{\mathrm{f}}: \mathbb{R}^{\mathrm{n}} \rightarrow \mathfrak{t}^{*}$ is the affine-linear map

$$
C_{f}: \mathbb{R}^{n} \rightarrow t^{*}, \quad\left(r_{1}, \ldots, r_{n}\right) \mapsto \Phi_{T}(f)+\sum_{i=1}^{n} r_{i} \operatorname{sign}\left(\left\langle\vec{v}, \lambda_{f}^{i}\right\rangle\right) \lambda_{f}^{i}
$$

which is proper when restricted to $\mathbb{R}_{\geq 0}^{n}$.

In particular each term is supported on a noncompact polyhedral cone, and much cancelation occurs to produce a compactly supported answer.

However, note that once one has computed $\mathrm{DH}(\mathrm{X}, \mathrm{T})$, one can use the restriction $1 \hookrightarrow T$ to compute the symplectic volume $\int_{X} e^{\omega}$ of $X$. There are two interesting subtleties in this restriction. One is that we can't pass from $T$ to the trivial group and then apply the theorem, because we lose the " $\mathrm{X}^{\top}$ isolated" condition. The other is that the total mass of $\mathrm{DH}(\mathrm{X}, \mathrm{T})$ can't be computed termby-term, since the mass of each term is infinite. (This latter problem can be fixed rather crudely by cutting $\mathfrak{t}^{*}$ with a half-space chosen to contain $\Phi_{\mathrm{T}}(\mathrm{X})$, or even just to contain the point at which one wishes to evaluate $\mathrm{DH}(\mathrm{X}, \mathrm{T})$.)

We mention that one can see from the above formula (or more directly) that if the kernel of T's action on $X$ is finite, i.e. at some (hence every) fixed point $f$ the $\left\{\lambda_{f}^{i}\right\}$ rationally span $t^{*}$, then $\mathrm{DH}(X, T)$ is Lebesgue measure times a piecewisepolynomial function, called the Duistermaat-Heckman function.

In their very influential paper [AB84], Atiyah and Bott (at the same time as Berline and Vergne in [BV84]) gave a formula for the integration of equivariant cohomology classes on a compact T-manifold, and showed that the DuistermaatHeckman formula is the special case of integrating the exponential of the equivariant symplectic form $\widetilde{\omega}:=\omega-\Phi_{\mathrm{T}}$. 
Theorem. [AB84, BV84] Let $\mathrm{X}$ be a compact oriented manifold, and $\alpha \in \mathrm{H}_{\mathrm{T}}^{*}(\mathrm{X})$, where $\mathrm{T}$ acts on $\mathrm{X}$ with isolated fixed points $\mathrm{X}^{\top}$, and as above let $\lambda_{1}^{\mathrm{f}}, \ldots, \lambda_{\mathrm{n}}^{\mathrm{f}}$ be the weights of $\mathrm{T}$ acting on the tangent space $\mathrm{T}_{\mathrm{f}} \mathrm{X}$ for each fixed point $\mathrm{f} \in \mathrm{X}^{\mathrm{T}}$.

Then the pushforward of $\alpha$ along the map $\mathrm{X} \rightarrow \mathrm{pt}$, denoted $\int_{\mathrm{X}} \alpha \in \mathrm{H}_{\mathrm{T}}^{*}(\mathrm{pt}) \cong$ $\operatorname{Sym}\left(\mathrm{T}^{*}\right)$, can be computed as

$$
\int_{X} \alpha=\sum_{f \in X^{\top}} \frac{\left.\alpha\right|_{f}}{\prod_{i=1}^{n} \lambda_{f}^{i}}
$$

where the right-hand side formally lives in the ring of fractions of the polynomial ring $\operatorname{Sym}\left(\mathrm{T}^{*}\right)$. Here $\left.\alpha\right|_{\mathrm{f}} \in \mathrm{H}_{\mathrm{T}}^{*}(\mathrm{f}) \cong \mathrm{H}_{\mathrm{T}}^{*}(\mathrm{pt})$ denotes the pullback of $\alpha$ along the $\mathrm{T}$-equivariant inclusion $\{\mathrm{f}\} \hookrightarrow \mathrm{X}$.

By definition, the equivariant cohomology ring $\mathrm{H}_{\mathrm{T}}^{*}(\mathrm{X})$ is the direct sum of the groups $H_{\top}^{i}(X)$. But the $A B / B V$ formula obviously extends to elements of the direct product $\prod_{i} H_{T}^{i}(X)$, such as $\exp (\widetilde{\omega})$. Then

$$
\int_{X} \exp (\widetilde{\boldsymbol{\omega}})=\sum_{x \in X^{\top}} \frac{\exp \left(-\Phi_{\mathrm{T}}(x)\right)}{\prod_{i=1}^{n} \lambda_{x}^{i}} .
$$

It is very tempting to Fourier transform term-by-term, turning $\exp \left(-\Phi_{\mathrm{T}}(\mathrm{f})\right)$ into $\delta_{\Phi_{\mathrm{T}}(\mathrm{f})}$, and the division by $\lambda_{f}^{i}$ into integration in the $\lambda_{f}^{i}$ direction. Making proper sense of this (fixing the constant of integration, one might say) requires the choice of $\vec{v}$ from the Duistermaat-Heckman theorem, and flipping of those weights for which $\left\langle\vec{v}, \lambda_{f}^{i}\right\rangle<0$. That done, the Duistermaat-Heckman theorem (in the [GLS88] form above) follows.

1.1. The basic formula. Hereafter we work in the algebro-geometric setting, largely to avoid questions relating to singularities of certain subsets of $X$; our localization theorem will thus be for equivariant Chow classes (see e.g. [Br97]). On the plus side, we will not require any smoothness assumption on $X$ itself; hereafter, throughout the paper, $X$ will always denote a projective scheme (except for a brief discussion in section 1.4). If the components of $X$ are of varying dimension, $\operatorname{dim} X$ means the maximum thereof.

Our main result is a different formula for the Duistermaat-Heckman measure, in which all the terms are themselves positive, and perforce compactly supported. Rather than maps of the orthant to $\mathfrak{t}^{*}$, the terms will be based on maps of the standard n-simplex $\left\{\vec{v} \in \mathbb{R}_{\geq 0}^{n}: \sum_{i} v_{i} \leq 1\right\}$ to $\mathfrak{t}^{*}$. 
Fix a one-parameter subgroup ${ }^{2} S: \mathbb{G}_{m} \rightarrow T$ such that $X^{S}=X^{\top}$. Then the Białynicki-Birula stratum [BB76], hereafter B-B stratum, $X_{f}$ is defined as the locally closed subset

$$
X_{f}:=\left\{x \in X: \lim _{z \rightarrow 0} S(z) \cdot x=f\right\}
$$

(considered with the reduced scheme structure, i.e., as a set). It is easy to see that $X=\coprod_{f \in X^{\top}} X_{f}$; this is called the B-B decomposition of $X$ (or more precisely, the pair $(X, S)$ ), and is the algebraic analogue of a Morse decomposition.

Unfortunately, this is usually not a stratification: the closure $\overline{X_{f}}$ is usually not a union of strata $\left\{X_{g}\right\}$ (one example to be given in section 1.2.2). Consequently, the combinatorics of the finite set $X^{\top}$ is much richer than just a partially ordered set (though it is ${ }^{3}$ that, by taking the transitive closure of " $g \geq f$ if $g \in \overline{X_{f}}$ "). Define $\overline{X_{f_{0}, \ldots, f_{k}}}$ inductively by

$$
\overline{X_{\emptyset}}:=X, \quad \overline{X_{f_{0}, \ldots, f_{k}}}:=\overline{\overline{X_{f_{0}, \ldots, f_{k-1}}} \cap X_{f_{k}}} .
$$

Call a nonrepeating sequence $\gamma=\left(f_{0}, \ldots, f_{k-1}\right)$ a closure chain if $\overline{X_{f_{0}, \ldots, f_{k}}}$ is nonempty, or equivalently, if $\overline{X_{f_{0}, \ldots, f_{k}}} \ni f_{k}$. Obviously this implies $f_{0}<f_{1}<$ $\ldots<f_{k}$, hence one can think of $\gamma$ as just a set, with the partial order on $X^{\top}$ "remembering" the order on $\gamma$.

It is easy to see that the set of closure chains forms a simplicial complex $\Delta(X, S)$ (meaning, any subset of a closure chain is itself one). Note that $\overline{X_{f_{0}, \ldots, f_{k-1}}} \cap X_{f_{k}}$ is itself a B-B stratum, namely $\left(\overline{X_{f_{0}, \ldots, f_{k-1}}}\right)_{f_{k}}$ in $\overline{X_{f_{0}, \ldots, f_{k-1}}}$, and hence connected when nonempty. We christen the set of all these subsets $\left\{\left(\overline{X_{f_{0}, \ldots, f_{k-1}}}\right)_{f_{k}}\right\}$ the iterated B-B filtration of $(X, S)$. Our most nontrivial result (proposition 3.1) about the complex $\Delta(X, S)$ is that it is equidimensional when $X$ is.

At this point, we can give a weak statement of our version of the DuistermaatHeckman formula. It will be in terms of the simplicial complex $\Delta(X, S)$, which does not depend on the projective embedding, and some coefficients $\left\{v_{\gamma} \in \mathbb{N}\right\}$

\footnotetext{
${ }^{2}$ While this $\mathrm{S}$, or rather its associated coweight, bears superficial similarity to the vector $\vec{v} \in \mathfrak{t}$ needed in the Duistermaat-Heckman theorem, its usage will be substantially different.

${ }^{3}$ This statement is quite nonobvious, actually, as it uses projectivity in a crucial way: otherwise one can glue two $\mathbb{P}^{1} \mathrm{~s}$ together, each one carrying the standard action of $\mathbb{G}_{\mathrm{m}}$, but each one's $\overrightarrow{0}$ glued to the other one's $\vec{\infty}$. Even smooth counterexamples have been constructed [Ju77]. In the smooth projective case, this statement appears in [BB76], and more generally can be proven with the technique of lemma 2.4 , though we will never use it directly.
} 
that do. We defer a precise definition of these coefficients until theorem 2 , and until then this is a sort of existence result.

Theorem 1. Let $\mathrm{X} \subseteq \mathbb{P V}$ be a subscheme invariant under the linear action of a torus $\mathrm{T}$ on the vector space $\mathrm{V}$. Assume that the fixed point set $\mathrm{X}^{\mathrm{T}}$ is finite, and let $\mathrm{S}: \mathbb{G}_{\mathrm{m}} \rightarrow \mathrm{T}$ be a one-parameter subgroup with $\mathrm{X}^{\mathrm{S}}=\mathrm{X}^{\mathrm{T}}$, with which to define the complex $\Delta(\mathrm{X}, \mathrm{S})$ of closure chains.

The longest a closure chain $\gamma$ may be is $1+\operatorname{dim} \mathrm{X}$ elements. (If $\mathrm{X}$ is equidimensional, then every maximal closure chain is indeed this long.) To each such closure chain $\gamma$, and depending on the projective embedding, there is associated a positive integer $\boldsymbol{v}_{\gamma}$, such that the Duistermaat-Heckman measure of $\mathrm{X}$ can be calculated as

$$
\left.\mathrm{DH}(\mathrm{X}, \mathrm{T})=\sum_{\gamma} v_{\gamma}\left(\mathrm{C}_{\gamma}\right)_{*} \text { (Lebesgue measure on the standard } \mathrm{n} \text {-simplex }\right)
$$

where $\mathrm{C}_{\gamma}$ is the unique affine-linear map $\mathbb{R}^{n} \rightarrow \mathfrak{t}^{*}$ taking the vertices of the standard simplex to $\left\{\Phi_{\mathrm{T}}(\mathrm{f}): \mathrm{f} \in \gamma\right\}$.

In particular, to determine the value at a point $\mathrm{p}$, we need only sum over those $\gamma$ such that $p$ lies in the convex hull of $\left\{\Phi_{\mathrm{T}}(\mathrm{f}): \mathrm{f} \in \gamma\right\}$.

Note that the Duistermaat-Heckman measure is not sensitive to components of lower dimension (geometric or embedded), so one may freely replace $\mathrm{X}$ by the union of its primary components of top dimension. It matters little because though this replacement may shrink $\Delta(X, S)$, it doesn't change the set of faces $\gamma$ summed over (as follows from corollary 3.1 and proposition 3.1).

As we explained after the Duistermaat-Heckman theorem, it is very tricky to turn their formula into one for the symplectic volume (or in the algebraic situation, the degree). Whereas here, since the individual terms have finite volume, we can forget the T-action term by term and obtain the formula $\operatorname{deg}(X)=\sum_{\gamma} v_{\gamma}$. We refine this sum in section 4.3.

\subsection{Examples of $\Delta(X, S)$.}

1.2.1. Flag manifolds. In the case that the $\mathrm{B}-\mathrm{B}$ decomposition is a stratification, then each nonempty $\left(\overline{X_{f_{0}, \ldots, f_{k-1}}}\right)_{f_{k}}$ is just $X_{f_{k}}$, and any chain $f_{0}<\ldots<f_{k}$ in the partial order is a closure chain. So the complex $\Delta(X, S)$ of closure chains is just 
the "order complex" of this poset $\mathrm{X}^{\top}$. Under a slightly stronger assumption, the theorem 1 here is an algebro-geometric version of theorem 1 in our earlier paper [Kn99], proven there for symplectic manifolds with no algebraicity condition.

Our inspiration for that formula was the case $X=G / P$ a generalized flag manifold, where the B-B decomposition is the Bruhat decomposition [Ak81] and the partial order is the Bruhat order. In this case the order complex is homeomorphic to a ball [BW82].

Each space $\Gamma(G / P ; \mathcal{O}(n))$ is an irreducible representation of $G$, so the exact formula for the T-equivariant Hilbert function (not just its asymptotics) is given by the Kostant multiplicity formula or the Littelmann path formula, one case of which was the Lakshmibai-Seshadri conjecture. As explained in [Kn99], the asymptotics of the Kostant and Lakshmibai-Seshadri formulae reproduce respectively the Duistermaat-Heckman theorem (this special case being Heckman's thesis) or theorem 1.

All the same analysis goes over to Schubert varieties inside flag manifolds, not just the flag manifolds themselves. The resulting formula for degrees of Schubert varieties is closely related to the one in [PS], and more distantly to the one in [Du03].

1.2.2. Toric varieties. Let $X$ be the complex toric variety associated to an integral polytope $P \subseteq \mathfrak{t}^{*}$. Each of the subsets $\overline{X_{f_{0}, \ldots, f_{k}}},\left(\overline{X_{f_{0}, \ldots, f_{k-1}}}\right)_{f_{k}}$ in the iterated B$\mathrm{B}$ filtration of $\mathrm{X}$ maps under the moment map $\Phi_{\mathrm{T}}$ onto a corresponding subset $\overline{P_{f_{0}, \ldots, f_{k}}},\left(\overline{P_{f_{0}, \ldots, f_{k-1}}}\right)_{f_{k}}$ of $P$, which will be easier to visualize.

The choice $S: \mathbb{G}_{m} \hookrightarrow T$ defines an "up" direction on $P$; the condition $X^{\top}=X^{S}$ says that each edge (hence each face) has a top vertex and a bottom vertex. Then $P_{f}$ (resp. $\overline{P_{f}}$ ) is the union of those open faces (resp. closed faces) of $P$ whose bottom vertex is $f$. If $P$ is a simple polytope, meaning that there are only $\operatorname{dim} P$ edges from each vertex (equivalently, $X$ has at worst orbifold singularities), then $P_{f}$ contains only one maximal face, but this is not always true: consider $\mathrm{P}$ an octahedron almost balanced on one corner, tilted over a little. Then the lowest of the four points on the equator has $\overline{\mathrm{P}_{\mathrm{f}}}=$ two triangles.

In this case $\Delta(X, S)$ is a well-known triangulation of $\mathrm{P}$ (a "pulling triangulation" by pulling the vertices starting from the bottom). More precisely, the maps $C_{\gamma}$ 
from the standard simplex to $\mathfrak{t}^{*}$ are embeddings, and their images in $\mathfrak{t}^{*}$ exactly cover P.

We illustrate in the case $\mathrm{P}$ a truncated right triangle, so $\mathrm{X}$ a Hirzebruch surface $F_{1}$, where one can already see the $\mathrm{B}-\mathrm{B}$ decomposition fail to be a stratification [BB76, example 1]. Pictured left-to-right are $\mathrm{P}$, its B-B decomposition, and $\Delta\left(X_{P}, S\right)$.
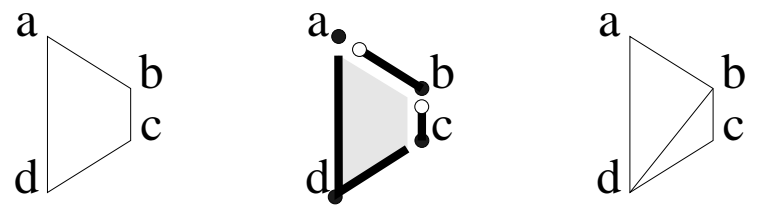

The closure of $X_{c}$ is not a union of strata (it intersects but doesn't contain $X_{b}$ ) and even though $b \in \overline{X_{c}}, a \in \overline{X_{b}}$, we don't have $a \in \overline{X_{c}}$ nor a triangle in $\Delta\left(X_{P}, S\right)$ containing $\{a, b, c\}$. The coefficient on $v_{\gamma}$ is the volume of the convex hull of $\Phi_{\mathrm{T}}(\gamma) \subseteq \mathrm{P}$, which partly motivated the choice of the letter $v$.

1.2.3. Stanley-Reisner schemes. In both the flag manifold and toric variety examples, the simplicial complexes $\Delta(\mathrm{X}, \mathrm{S})$ were very special: they were homeomorphic to balls. In this section we show that in the general case, any simplicial complex may arise. The following examples do not provide interesting applications of theorem 1, but are good for testing one's intuition about closure chains.

Let $\Delta$ be an arbitrary simplicial complex on the vertex set $1, \ldots, n$, and let $V=\mathbb{A}^{n}$. To each face $F$ in $\Delta$, we associate the coordinate projective subspace $X(F) \subseteq \mathbb{P} V$ that uses only those coordinates. Then let $X(\Delta)=\cup_{F \in \Delta} X(F)$ be the union of those coordinate projective subspaces. This $X(\Delta)$ is the (projective) Stanley-Reisner scheme of $\Delta$, whose coordinate ring is the (homogeneous) Stanley-Reisner ring of $\Delta$. It is invariant under the torus $T$ that scales each coordinate independently.

Let $\Phi_{S}:\{1, \ldots, n\} \hookrightarrow \mathbb{Z}$ be strictly increasing. (Really the important condition is injectivity, but by permuting $1, \ldots, n$ we can obtain this convenient stronger condition.) Then there is a corresponding action of $\mathbb{G}_{m}$ on $\mathbb{P V}$, by

$$
S(z) \cdot\left[x_{1}, \ldots, x_{n}\right]:=\left[z^{\Phi_{S}(1)} x_{1}, \ldots, z^{\Phi_{S}(i)} x_{i}, \ldots, z^{\Phi_{S}(n)} x_{n}\right]
$$


which fixes $X(\Delta)$. The condition that $\Phi_{S}$ is injective says that the only $S$-fixed points on $\mathbb{P V}$ are the coordinate points (actually it is enough that $\Phi_{S}(f) \neq \Phi_{S}(g)$ for each edge $\{f, g\} \in \Delta$ ).

Proposition 1.1. Let $\Delta, X(\Delta), \Phi_{S}, S$ be as above. Then the associated simplicial complex of closure chains is just $\Delta$. In particular, every finite simplicial complex arises in this way.

Proof. Identify the fixed points $\{[0, \ldots, 0,1,0, \ldots, 0]\}$ with $1, \ldots, n$. Then it is easy to show that

$$
X(\Delta)_{i}=\overline{X(\Delta)_{i}} \cap\left\{\left[x_{1}, \ldots, x_{n}\right]: x_{i} \neq 0\right\} \quad \text { where } \quad \overline{X(\Delta)_{i}}=\bigcup_{F \in \Delta, \min (F)=i} X(F) .
$$

(This would be more irritating to state without having first made $\Phi_{\mathrm{S}}$ strictly increasing.) From this, one can show inductively that

$$
\overline{X(\Delta)_{f_{0}, \ldots, f_{k}}}=\bigcup\left\{X(F): F \in \Delta, F=\left\{f_{0}, \ldots, f_{k}, \text { larger numbers }\right\}\right\}
$$

so the left side is nonempty iff $\left\{f_{0}, \ldots, f_{k}\right\}$ is the initial string of a face of $\Delta$, i.e. iff it is a face of $\Delta$.

When theorem 1 is applied to $X(\Delta)$, each mysterious coefficient $\nu_{\mathrm{F}}$ is just 1 , the degree of the projective variety $\mathrm{X}(\mathrm{F})$.

1.2.4. Some tricky behavior. We first mention a geometric subtlety of the definition of closure chain. Plainly $\overline{X_{f_{0}, \ldots, f_{k}}}$ is contained in $\overline{X_{f_{0}, \ldots, f_{k-1}}} \cap \overline{X_{f_{k}}}$, since it is defined as the closure of $\overline{X_{f_{0}, \ldots, f_{k-1}}} \cap X_{f_{k}}$. But it can be strictly smaller, as we will show by example in a moment. One can show that if $\overline{X_{f_{0}, \ldots, f_{k}}}$ had instead been defined as $\overline{X_{f_{0}, \ldots, f_{k-1}}} \cap \overline{X_{f_{k}}}$, then the (similarly larger) complex of closure chains would be a "clique complex", meaning, the largest simplicial complex with a given set of 1-faces. For example, order complexes of posets are clique complexes, where the 1 -faces $\{a, b\}$ specify comparability of $a$ and $b$.

The smallest simplicial complex that isn't a clique complex is a hollow triangle (the clique complex would be the solid triangle). The corresponding StanleyReisner scheme is $X=\{[a, b, c]: a b c=0\} \subseteq \mathbb{P}^{2}$. Taking $S(z) \cdot[a, b, c]:=$ $\left[a, z b, z^{2} c\right]$, the B-B strata are

$$
X_{[1,0,0]}=\{[1, b, 0]\} \cup\{[1,0, c]\}, \quad X_{[0,1,0]}=\{[0,1, c]\}, \quad X_{[0,0,1]}=\{[0,0,1]\}
$$


and the complex of closure chains is the desired hollow triangle. In this example, we see the claimed geometric subtlety at $\overline{X_{[1,0,0],[0,1,0]}}=\{[0,1,0]\}$, contrasted with the strictly larger $\overline{X_{[1,0,0]}} \cap \overline{X_{[0,1,0]}}=\{[0,1,0],[0,0,1]\}$.

Another interesting (for other reasons) example in the plane is $X=\{[a, b, c]$ : $\left.\mathrm{b}\left(\mathrm{ac}-\mathrm{b}^{2}\right)=0\right\}$, invariant under the same $\mathrm{S}$ as above, and we take $\mathrm{T}=\mathrm{S}$. This has two $S$-fixed points $\{[1,0,0],[0,0,1]\}$, and the complex of closure chains is an interval. (The unique $v_{\gamma}$ turns out to be 3 , the degree of $X$.) Whereas the geometry of $X$ - a line union a conic, meeting transversely at the two S-fixed points - would seem to suggest that the more appropriate complex would be an oval, made with two intervals glued together at both ends (their $v_{\gamma} \mathrm{s}$ being 1 for the line and 2 for the conic).

However, that is not a simplicial complex (in which faces are determined by their set of vertices), but falls under the slightly more general notion of simplicial poset. We did not need this richer notion to formulate theorem 1, but we will use it in the K-theory version $[\mathrm{Kn}]$, based on ideas from [Kn06].

Having just described a simplicial complex (the clique complex above) that is slightly larger than we need, the reader may wonder whether the complex $\Delta(X, S)$ might still be larger than necessary. One sign that the complex is a good one is that the coefficients $v_{\gamma}$ in theorem 1 are strictly positive, so no term may be omitted. Another is that in the toric variety case discussed in the previous section, the supports of the terms are disjoint, so the $v_{\gamma}$ can't even be adjusted to leave some term out. Another indication of $\Delta(X, S)$ 's minimality will come in proposition 3.1.

1.2.5. A Bott-Samelson manifold. Bott-Samelson manifolds provide examples of $\Delta(\mathrm{X}, \mathrm{S})$ that are not homeomorphic to balls, despite $\mathrm{X}$ being irreducible. Consider the variety $X \subset \operatorname{Gr}_{1}\left(\mathbb{C}^{3}\right) \times \operatorname{Gr}_{2}\left(\mathbb{C}^{3}\right) \times \mathrm{Gr}_{1}\left(\mathbb{C}^{3}\right)$ of triples of subspaces, with inci-

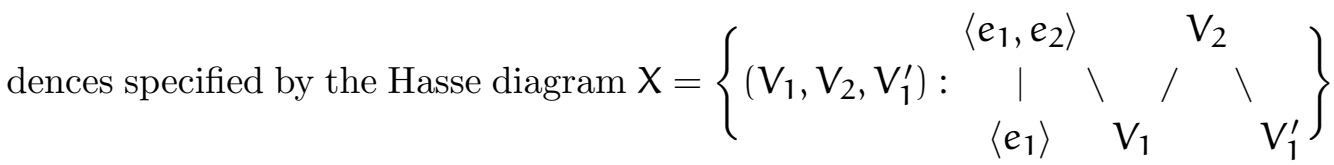
where $\left\{e_{1}, e_{2}, e_{3}\right\}$ denote the standard basis of $\mathbb{C}^{3}$.

This is the "1-2-1 Bott-Samelson manifold"; one way to think of it is as a walk from the base flag $\left(\left\langle e_{1}\right\rangle,\left\langle e_{1}, e_{2}\right\rangle\right)$ to other flags, by changing the line, then the plane, then the line again. It carries an action of the diagonal matrices $\mathrm{T}$ inside 
$\mathrm{GL}_{3}(\mathbb{C})$ (indeed, of the upper triangulars). There are $2^{3}=8 \mathrm{~T}$-fixed points, in which $V_{1}, V_{2}, V_{1}^{\prime}$ are coordinate subspaces. They are indexed by subsets of the word 121, where a letter is included if the corresponding subspace is different from the previous choice.

The Bott-Samelson is a blowup of the flag manifold, via the map $\left(\mathrm{V}_{1}, \mathrm{~V}_{2}, \mathrm{~V}_{1}^{\prime}\right) \mapsto$ $\left(0<V_{1}^{\prime}<V_{2}<\mathbb{C}^{3}\right)$. The exceptional locus will turn out to be $\overline{X_{1--}}$. As such, $X^{\prime}$ 's moment polytope is a subpolytope of that of the flag manifold, and we draw it below:

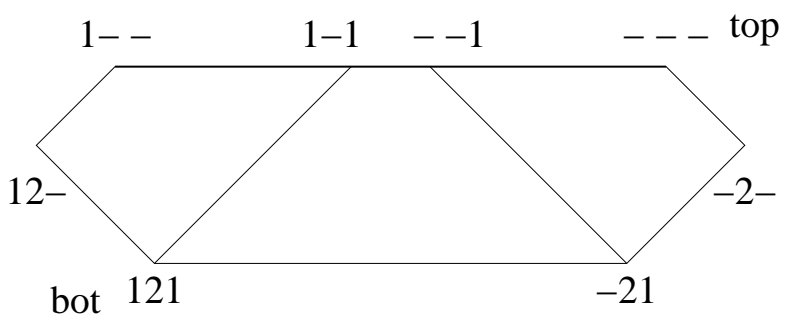

Our one-parameter subgroup $S: \mathbb{C}^{\times} \rightarrow \mathrm{T}$ will be $S(z)=\operatorname{diag}\left(1, z, z^{2}\right)$. Then the top (most repellent) point is $\left(\left\langle e_{1}\right\rangle,\left\langle e_{1}, e_{2}\right\rangle,\left\langle e_{1}\right\rangle\right)$, and the bottom is $\left(\left\langle e_{2}\right\rangle,\left\langle e_{2}, e_{3}\right\rangle,\left\langle e_{3}\right\rangle\right)$.

The closures of the B-B strata are easy to compute:

$$
\begin{gathered}
\overline{\mathrm{X}_{121}}=\mathrm{X} \\
\overline{\mathrm{X}_{-21}}=\left\{\mathrm{V}_{1}=\left\langle e_{1}\right\rangle\right\}, \quad \overline{\mathrm{X}_{12-}}=\left\{\mathrm{V}_{1}=\mathrm{V}_{1}^{\prime}\right\}, \quad \overline{\mathrm{X}_{-2-}}=\left\{\mathrm{V}_{1}=\mathrm{V}_{1}^{\prime}=\left\langle e_{1}\right\rangle\right\} \\
\overline{\mathrm{X}_{1--}}=\left\{\mathrm{V}_{2}=\left\langle e_{1}, e_{2}\right\rangle\right\}, \quad \overline{\mathrm{X}_{--1}}=\overline{\mathrm{X}_{1--}} \cap \overline{\mathrm{X}_{-21}}, \quad \overline{\mathrm{X}_{1-1}}=\overline{\mathrm{X}_{1--}} \cap\left\{\mathrm{V}_{1}^{\prime}=\left\langle e_{1}\right\rangle\right\} \\
\overline{\mathrm{X}_{---}}=\left\{\mathrm{V}_{1}=\mathrm{V}_{1}^{\prime}=\left\langle e_{1}\right\rangle, \mathrm{V}_{2}=\left\langle e_{1}, e_{2}\right\rangle\right\}
\end{gathered}
$$

To check that each $\bar{X}_{f}$ is as claimed, note that it is T-invariant, and has the right local behavior at $f$ (an easy tangent space calculation on $X_{f}$, which is smooth because $X$ is smooth). So far this guarantees that the B-B stratum $X_{f}$ is open inside the purported $\bar{X}_{f}$. But then note that each $\bar{X}_{f}$ is irreducible, hence is the closure of $\mathrm{X}_{\mathrm{f}}$.

Note that this is not a stratification, as $\overline{X_{12-}} \not \supset \overline{X_{1--}}$. Rather,

$$
\overline{\mathrm{X}_{12-, 1--}}=\left\{\mathrm{V}_{1}=\mathrm{V}_{1}^{\prime}, \mathrm{V}_{2}=\left\langle\mathrm{e}_{1}, \mathrm{e}_{2}\right\rangle\right\}
$$

This is the diagonal of $\overline{X_{1--}} \cong\left(\mathbb{C P}^{1}\right)^{2}$, whereas $\overline{X_{--1}}, \overline{X_{1-1}}$ are its two axes. 
It remains to compute $\Delta(X, S)$. Because $\overline{X_{121}}=X$, the point 121 will be a "cone point", meaning that $\Delta(X, S)$ is a cone from that point. Put another way, it is uninteresting, so let's leave it out for now.

Since the top point - - - is in every stratum closure, it also will be a cone point. (While bot being a cone point occurs whenever $X$ is irreducible, top being a cone point is much more a surprise; top is not a cone point for most toric varieties, such as the Hirzebruch surface in section 1.2.2.)

The complex $\Delta(\mathrm{X}, \mathrm{S})$ is then the double cone (from 121 and - - ) on the 1-complex depicted below:

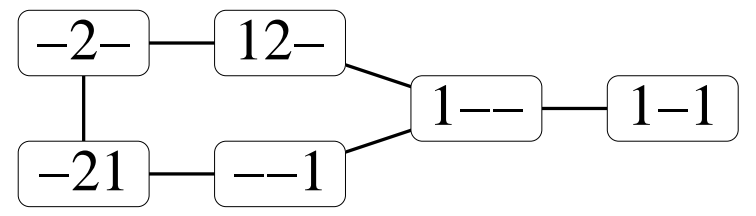

This $\Delta(\mathrm{X}, \mathrm{S})$ is not homeomorphic to a ball, though it is Cohen-Macaulay. We do not know how often this latter conclusion holds.

If the opposite B-B stratification is used $(z \rightarrow \infty$ rather than $z \rightarrow 0$, switching the roles of bot and top), it turns out that there is only the one cone point - - This $\Delta\left(X, S^{-1}\right)$, depicted below without its cone point, is again Cohen-Macaulay though not homeomorphic to a ball.

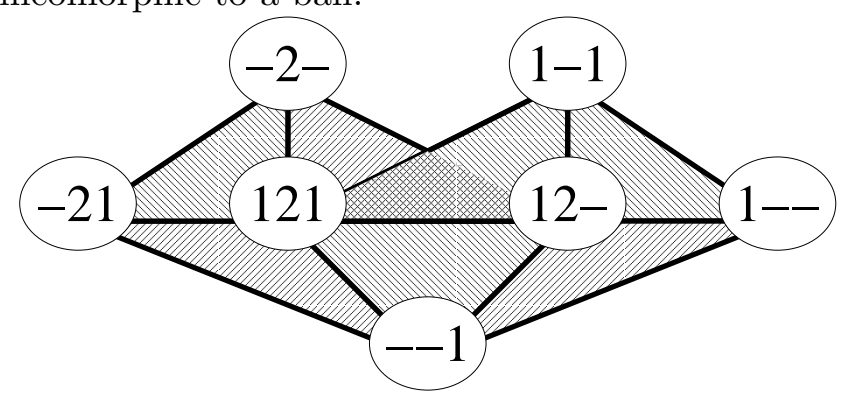

1.2.6. The punctual Hilbert scheme of four points in $\mathbb{C}^{2}$. The Hilbert scheme of $\mathrm{n}$ points in the complex plane is defined very concretely as the set of ideals in $\mathbb{C}[x, y]$ of codimension $n$. It is, miraculously, smooth (Fogarty's theorem) and has received a lot of attention recently, such as in our reference [Ha02].

The subscheme in which the $\mathrm{n}$ points all sit at the origin is even more concrete: each ideal contains $(x, y)^{n}$, so can be considered an ideal in $\mathbb{C}[x, y] /(x, y)^{n}$, and 
hence a point in the Grassmannian $\operatorname{Gr}_{\left(\begin{array}{c}n \\ 2\end{array}\right)}\left(\mathbb{C}[x, y] /(x, y)^{n}\right)$. This subscheme turns out to be irreducible (another miracle), though not smooth.

The $T$ acting is the diagonal matrices from $\mathrm{GL}_{2}(\mathbb{C})$, which acts on the ring and hence on the set of ideals. The fixed points are the ideals I generated by monomials, and are indexed by partitions of $n$ as follows: the set of pairs $\{(a, b)$ : $\left.x^{\mathrm{a}} \mathrm{y}^{\mathrm{b}} \notin \mathrm{I}\right\} \subset \mathbb{N}^{2}$ is automatically a partition.

We draw the T-moment polytope for this action on the punctual Hilbert scheme of 4 points at the origin of the plane, labeling each vertex by its partition. We put edges to indicate the T-invariant $\mathbb{P}^{1} \mathrm{~s}$, though we won't make direct use of them.

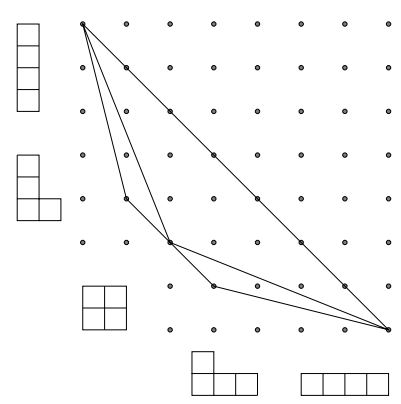

From Northwest to Southeast, these vertices are the ideals $\left(y^{4}, x\right),\left(y^{3}, x y, x^{2}\right)$, $\left(y^{2}, x^{2}\right),\left(y^{2}, x y, x^{3}\right)$, and $\left(y, x^{4}\right)$.

Let $S: \mathbb{C}^{\times} \rightarrow \mathrm{GL}(2)$ be the one-parameter subgroup $z \mapsto \operatorname{diag}\left(z, z^{2}\right)$, so $\left(y^{4}, x\right)$ is the top and $\left(y, x^{4}\right)$ the bottom. We now describe the closures of the B-B strata on the Hilbert scheme: ${ }^{4}$

$$
\begin{gathered}
\overline{X_{\left(y^{4}, x\right)}}=\left\{\left(y^{4}, x\right)\right\} \quad \overline{X_{\left(y^{3}, x y, x^{2}\right)}}=\left\{\left(y^{4}, x y, x^{2}, A x+B y^{3}\right)\right\} \\
\overline{X_{\left(x^{2}, y^{2}\right)}}=\left\{\left(y^{4}, x y^{2}, x^{2}, A x+B y^{2}+C x y+D y^{3}, A x y+B y^{3}\right)\right\} \\
\overline{X_{\left(y^{2}, x y, x^{3}\right)}}=\left\{\left(y^{3}, y^{2} x, y x^{2}, x^{3}, B x^{2}+C x y+D y^{2}, E x^{2}+F x y+G y^{2}\right)\right\} \\
\overline{X_{\left(y, x^{4}\right)}}=X
\end{gathered}
$$

where not both of $A, B$ are zero, and $(B, C, D),(E, F, G)$ are linearly independent. As these strata are not smooth, these claims are harder to check, but we do not take space to do so here.

${ }^{4}$ The obvious terminology for these is "Gröbner basins". 
Since $X$ is irreducible, bot $=\left(y, x^{4}\right)$ is a cone point in the complex $\Delta(X, S)$, but top is not one as top $\notin \overline{X_{\left(y^{2}, x y, x^{3}\right)}}$. The complex is pictured below, without the cone point:

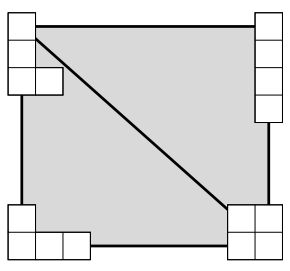

1.3. The coefficients $v_{\gamma}$. We now give a recurrence on a family $\left\{v(Z)_{\left(f_{0}, \ldots, f_{k}\right), Y}\right\}$ of natural numbers, where $Y$ is an irreducible component of $\overline{Z_{f_{0}, \ldots, f_{k}}}$ of codimension $k$ in $Z$, in order to give a quick definition of the $\left\{v_{\gamma}\right\}$.

If $\mathrm{X}$ is irreducible, it has a unique open B-B stratum, and we denote the fixed point in that stratum $\min (\mathrm{X})$. Lemma 2.4 then implies the following: there exists a unique $T$-invariant hyperplane section of $X$ supported on $X \backslash X \min (X)$. It is at this point that the projective embedding of $\mathrm{X}$ is finally felt: the scheme structure on this hyperplane section gives multiplicities on the components of $X \backslash X_{\min (X)}$, and these multiplicities are building blocks in the definition of the $\left\{v_{\gamma}\right\}$.

Theorem 2. Let $Z$ vary over the class of $\mathrm{T}$-invariant subschemes of $\mathbb{P} V$ with $Z^{\top}$ isolated, and $\mathrm{Y}$ over the irreducible components of $\overline{Z_{\mathrm{f}_{0}, \ldots, \mathrm{f}_{\mathrm{k}}}}$ of codimension $\mathrm{k}$ in $\mathrm{Z}$. (There will only exist such $\mathrm{Y}$ if $\left(\mathrm{f}_{\mathrm{O}}, \ldots, \mathrm{f}_{\mathrm{k}}\right)$ is a closure chain, and usually not even then.)

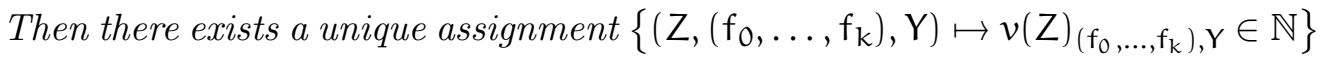
satisfying the following conditions:

(1) For $0 \leq \mathfrak{j} \leq \mathrm{k}$ (though we will only use $\mathfrak{j}=0,1)$ :

$$
v(Z)_{\left(f_{0}, \ldots, f_{j}, \ldots, f_{k}\right), Y}=\sum_{Y_{j} \subseteq Z_{f_{0}, \ldots, f_{j}}, Y_{j} \supseteq Y} v(Z)_{\left(f_{0}, \ldots, f_{j}\right), Y_{j}} v\left(Y_{j}\right)_{\left(f_{j}, \ldots, f_{k}\right), Y}
$$

where the sum is over components $Y_{j}$ of $\overline{Z_{f_{0}, \ldots, f_{j}}}$ of codimension $j$ in $Z$.

(2) $v(Z)_{(\min (Z)), Y}$ is the multiplicity of $\mathrm{Y}$ as a component of $\mathrm{Z}$.

(3) If $Z$ is reduced and irreducible, and $Y$ is a component of $Z \backslash Z \min (Z)$, then $v(Z)_{(\min (Z), \min (\mathrm{Y})), \mathrm{Y}}$ is the multiplicity of $\mathrm{Y}$ in the $\mathrm{T}$-invariant hyperplane section of $Z$ supported on $Z \backslash Z \min (Z)$.

The coefficients $v_{\gamma}$ from theorem 1 can be calculated as $v_{\gamma=\left(f_{0}, \ldots, f_{\operatorname{dim} x}\right)}=v(X)_{\gamma,\left\{f_{\operatorname{dim} x}\right\}}$. 
Unfortunately these multiplicities $v(Z)_{(\min (Z), \min (\mathrm{Y})), \mathrm{Y}}$ can be very difficult to compute in examples, particularly if $Z$ is singular at $\min (Y)$. In section 4.2 we prove some linear relations on the $\left\{v_{\gamma}\right\}$ to help constrain them.

1.4. Integrating more general classes. One of the advances of [AB84, BV84] was to give a formula for integrating more general classes than just $\exp (\widetilde{\boldsymbol{\omega}})$. When $\mathrm{X}$ is a symplectic manifold, one application of this equivariant integration is to perform ordinary integration on symplectic/GIT quotients $X / / \mathrm{p} T$ of $X$. Recall [Ki86] that for $p$ a regular value of the moment map $\Phi_{\mathrm{T}}$, there is a surjective Kirwan map $\mathrm{\kappa}: \mathrm{H}_{\mathrm{T}}^{*}(\mathrm{X}) \rightarrow \mathrm{H}^{*}(\mathrm{X} / / \mathrm{p} \mathrm{T})$, whose kernel can be computed by computing integrals on $\mathrm{X} / / \mathrm{p} \mathrm{T}$. That can be done as follows:

Proposition 1.2. [Gu94] Assume the setup $\mathrm{X}, \Phi_{\mathrm{T}}$ of the Duistermaat-Heckman theorem, and let $\mathrm{p} \in \Phi_{\mathrm{T}}(\mathrm{X})$ be a regular value, so the symplectic reduction $\mathrm{X} / /_{\mathrm{p}} \mathrm{T}$ is an orbifold with its own symplectic form $\omega_{\mathrm{p}}$. Let $\alpha \in \mathrm{H}_{\mathrm{T}}^{*}(\mathrm{X})$.

Then the Fourier transform of $\int_{X} \alpha \exp (\widetilde{\boldsymbol{\omega}})$ is a measure supported on $\Phi_{\mathrm{T}}(\mathrm{X})$, equal to Lebesgue measure times a polynomial in a neighborhood of $\mathrm{p}$, whose value at $\mathrm{p}$ is $\int_{\mathrm{X} / \mathrm{p} \mathrm{T}} \mathrm{K}(\alpha) \exp \left(\omega_{\mathrm{p}}\right)$. In particular, if $\operatorname{deg}(\alpha)=\operatorname{dim}(\mathrm{X} / / \mathrm{p} T)$ then the Fourier transform is a piecewise constant function (times Lebesgue measure) whose value at $\mathrm{p}$ is $\int_{\mathrm{X} / / \mathrm{p} \mathrm{T}} \mathrm{K}(\alpha)$.

This is also used as [GM06, theorem 3.2].

The case $\alpha \in \mathrm{H}_{\mathrm{T}}^{*}(\mathrm{pt})$, studied in [GS95], is already interesting, even though $\int \alpha \exp (\widetilde{\boldsymbol{\omega}})=\alpha \int \exp (\widetilde{\boldsymbol{\omega}})$. In this case, to compute the Fourier transform, we can first compute the D-H measure and then apply the differential operator $\widehat{\alpha}$. that is Fourier dual to multiplication by $\alpha$. Since $\mathrm{DH}(X, T)$ is a piecewise-polynomial times Lebesgue measure, this distribution can be very complicated along the breaks; it will thus be very convenient for us that the proposition above only requires that we understand it at generic $\mathrm{p}$.

We now give a formula for these distributions at generic $p$, in the same terms as in theorem 1 , and afterward discuss the case of general $\alpha \in \mathrm{H}_{\mathrm{T}}^{*}(\mathrm{X})$, or really $\alpha \in A_{*}^{\top}(X)$.

Given a list $\underline{v}:=\left(v_{1}, \ldots, v_{n}\right)$ of vectors in $V$ and a number $k \in \mathbb{N}$, define a partial fractions schema as an injection $\sigma:\{1, \ldots, k\} \hookrightarrow\{1, \ldots, n\}$ such that 
$\left(v_{j}: j \neq \sigma(1), \ldots, \sigma(k)\right)$ spans $V$ (so in particular $\left.k \leq n-\operatorname{dim} V\right)$, and that for each $i=1, \ldots, k$, one has that $\sigma(i)$ is in the lex-first basis in $\left(v_{j}: j \neq \sigma(1), \ldots, \sigma(i-1)\right)$.

Given also a subset $M \subseteq \underline{v}$ with $n-k$ elements, define the partial fractions k-tensor $\tau_{\underline{v}, M}$ as the sum

$$
\tau_{\underline{v}, M}=\sum_{\substack{\sigma \\ \sigma(\{1, \ldots, k\})=\underline{v} \backslash M}} v^{\sigma(1)} \otimes \cdots \otimes v^{\sigma(k)} \in\left(\mathrm{V}^{*}\right)^{\otimes k}
$$

over all partial fractions schemata $\sigma$ whose image is the complement of $M$, where $v^{\sigma(i)} \in \mathrm{V}^{*}$ denotes the dual basis element to $v_{\sigma(\mathfrak{i})}$ in that lex-first basis in $\left\{v_{j}: \mathfrak{j} \notin\right.$ $\sigma(1, \ldots, i-1)\}$.

Finally, given a pair $\left(\gamma^{\prime} \subseteq \gamma\right)$ of closure chains, with $\gamma^{\prime}=\left(f_{0}, \ldots, f_{k}\right)$ and $|\gamma|=1+\operatorname{dim} X$, let $\tau_{\gamma^{\prime}, \gamma}$ denote the partial fractions k-tensor $\tau_{\left(v_{i}\right), M}$ where $\mathrm{V}=\mathrm{T}^{*} \oplus \mathbb{Z} \mathrm{D}, v_{\mathrm{i}}=\mathrm{D}+\Phi_{\mathrm{T}}\left(\mathrm{f}_{\mathrm{i}}\right)$, and $\mathrm{M}=\gamma^{\prime}$.

Theorem 3. Assume the setup of theorem 1, and associate the same positive integers $v_{\gamma}$ to closure chains of length $1+\operatorname{dim} \mathrm{X}$. To each closure chain $\gamma^{\prime}$ with $1+\operatorname{dim} \mathrm{X}-\mathrm{k}$ elements, we associate the tensor

$$
v_{\gamma^{\prime}}:=\sum_{\gamma \supseteq \gamma^{\prime}} v_{\gamma} \tau_{\gamma^{\prime}, \gamma}, \quad \gamma \in \Delta(\mathrm{X}, \mathrm{S}),|\gamma|=1+\operatorname{dim} \mathrm{X}
$$

where $\tau_{\gamma^{\prime}, \gamma}$ is the partial fractions tensor defined above.

Let $\left(\alpha_{1}, \ldots, \alpha_{k}\right) \in \mathrm{T}^{*} \cong \mathrm{A}_{\mathrm{T}}^{1}(\mathrm{pt})$ be a list of $\mathrm{T}$-weights, so $\prod_{i=1}^{\mathrm{k}} \alpha_{\mathrm{i}} \in \mathrm{A}_{\mathrm{T}}^{\mathrm{k}}(\mathrm{pt})$ is a homogeneous class of degree $\mathrm{k}$. Then near any point of $\mathrm{p} \in \mathfrak{t}^{*}$ in general position, the Fourier transform of multiply-by- $\alpha$, applied to $\mathrm{DH}(\mathrm{X}, \mathrm{T})$, can be calculated as

$$
\sum_{\substack{\gamma^{\prime} \in \Delta(X, S) \\\left|\gamma^{\prime}\right|=1+\operatorname{dim} X-k}}\left(v_{\gamma^{\prime}} \cdot\left(\alpha_{1} \otimes \cdots \otimes \alpha_{k}\right)\right)\left(C_{\gamma^{\prime}}\right)_{*}
$$

(Lebesgue measure on the standard $(\mathrm{n}-\mathrm{k})$-simplex)

where $\mathrm{C}_{\gamma}$ is the unique affine-linear map $\mathbb{R}^{n-k} \rightarrow \mathfrak{t}^{*}$ taking the vertices of the standard simplex to $\left\{\Phi_{\mathrm{T}}(\mathrm{f}): \mathrm{f} \in \gamma^{\prime}\right\}$. ("General position" means here that $\mathrm{p}$ does not lie in the convex hull of fewer than $1+\operatorname{dim} \mathrm{T}$ elements of $\Phi_{\mathrm{T}}\left(\mathrm{X}^{\mathrm{T}}\right)$.)

In particular, to determine the value at a point $\mathrm{p}$ in general position, we need only sum over those $\gamma^{\prime}$ such that $p$ lies in the convex hull of $\left\{\Phi_{\mathrm{T}}(\mathrm{f}): f \in \gamma^{\prime}\right\}$. 
The next step beyond $\alpha \in H_{\mathrm{T}}^{*}(\mathrm{pt})$ is $\alpha$ of the form $\sum_{i} \alpha_{i}\left[X_{i}\right]$, where $\alpha_{i} \in$ $\mathrm{H}_{\mathrm{T}}^{*}(\mathrm{pt})$ and each $\mathrm{X}_{\mathrm{i}} \subseteq \mathrm{X}$ is a $\mathrm{T}$-invariant oriented submanifold. Then

$$
\int_{X} \alpha \exp (\widetilde{\boldsymbol{\omega}})=\int_{X}\left(\sum_{i} \alpha_{i}\left[X_{i}\right]\right) \exp (\widetilde{\boldsymbol{\omega}})=\sum_{i} \alpha_{i} \int_{X}\left[X_{i}\right] \exp (\widetilde{\boldsymbol{\omega}})=\sum_{i} \alpha_{i} \int_{X_{i}} \exp (\widetilde{\boldsymbol{\omega}}) .
$$

The Chow setting that we work in for the rest of the paper is closer to equivariant homology than cohomology, and has a very appealing feature [Br97, theorem 2.1]: every class $\alpha \in A_{*}^{\top}(X)$ is of the form $\sum_{i} \alpha_{i} \cap\left[X_{i}\right]$, for $\left\{X_{i}\right\}$ some T-invariant subvarieties. Since theorem 3 makes no smoothness assumption, it can be applied to the $X_{i}$ individually.

We admit here that the statement of theorem 3 is probably too unwieldy to see much direct use. We included it mainly to emphasize that, thanks to [Br97, theorem 2.1], an analogue of theorem 1 for general classes $\alpha \in A_{*}^{\top}(X)$ follows in some sense automatically from the $\alpha=1$ case already treated.

\section{BaCkground on D-H measures And B-B Decompositions}

In this section we assemble some results, well-known to the experts, on Duistermaat-Heckman measures and Białynicki-Birula decompositions, making little claim to originality. The closest reference we could find for the B-B results was [He81].

2.1. D-H measures and equivariant Chow theory. We first recast the calculation of the $\mathrm{D}-\mathrm{H}$ measure of $\mathrm{X}$ in terms of the equivariant Chow class of the affine cone $\widehat{X}$. This is desirable largely in that it lets us trade $X$ 's multiple fixed points for a single fixed point at the origin (though even when $X$ is smooth, $\widehat{X}$ won't be, so we can't use arguments that depend on smoothness). The base $\operatorname{ring} A_{\mathrm{T}}^{*}(p t) \cong \operatorname{Sym}\left(\mathrm{T}^{*}\right) \cong \mathrm{H}_{\mathrm{T}}^{*}(p t)$ is the same, and the intuition and results for Chow classes are well developed. Our references for equivariant Chow theory are [Br97, Br98].

All of our equivariant Chow classes will live on vector spaces. When $Z \subseteq W$ for $W$ a vector space, we will write $[Z \subseteq W]$ for the corresponding class in $A_{\mathrm{T}}^{*}(\mathrm{~W}) \cong \operatorname{Sym}\left(\mathrm{T}^{*}\right)$. Usually $W$ will be our ambient space $\mathrm{V}$, and then we will denote $[\mathrm{Z} \subseteq \mathrm{V}]$ simply by $[\mathrm{Z}]$.

The only facts we need about equivariant Chow classes are these trivial generalizations from ordinary Chow theory: 
Proposition 2.1. Let a torus $\mathrm{U}$ act on a vector space $\mathrm{V}$, preserving a subscheme $\mathrm{Y}$ and a hyperplane $\mathrm{H}=\{\mathrm{b}=0\}$, with $\lambda \in \mathrm{U}^{*}$ the $\mathrm{U}$-weight on the line $\mathrm{V} / \mathrm{H}$. For any $\mathrm{U}$-invariant subscheme $\mathrm{Z} \subseteq \mathrm{V}$, let $[\mathrm{Z}] \in \mathrm{A}_{\mathrm{U}}^{*}(\mathrm{~V})$ denote the associated equivariant Chow class.

- If $\mathrm{Y}$ contains no components in $\mathrm{H}$, i.e. if $\mathrm{b}$ is not a zero divisor on $\mathrm{Y}$, then $[\mathrm{H} \cap \mathrm{Y}]=[\mathrm{H}][\mathrm{Y}]=\lambda[\mathrm{Y}]$.

Conversely, if $\mathrm{Y} \subseteq \mathrm{H}$ then $[\mathrm{Y}]=[\mathrm{H}][\mathrm{Y} \subseteq \mathrm{H}]$, and $=[\mathrm{H}][\mathrm{Y} \times \mathrm{L}]$ where $\mathrm{L}$ is a $\mathrm{U}$-invariant complement in $\mathrm{V}$ to $\mathrm{H}$.

- If $\left\{\mathrm{Y}_{\mathrm{i}}\right\}$ are the top-dimensional geometric components of $\mathrm{Y}$, occurring with multiplicities $\left\{\mathrm{m}_{i} \in \mathbb{N}\right\}$, then $[\mathrm{Y}]=\sum_{i} \mathrm{~m}_{i}\left[\mathrm{Y}_{i}\right]$.

- If there exists a closed subscheme $\mathrm{F} \subseteq \mathrm{V} \times \mathrm{S}$ whose projection to the connected base $\mathrm{S}$ is flat, and whose fibers are $\mathrm{U}$-invariant subschemes two of whom are $\mathrm{Y}$ and $\mathrm{Y}^{\prime}$, then $[\mathrm{Y}]=\left[\mathrm{Y}^{\prime}\right]$.

The condition in the first is very easy to check when $Y$ is reduced and irreducible, and the second lets one reduce to that case. Joseph described this same recursion on his polynomials in [Jo97].

In fact we will work with the $\left(T \times \mathbb{G}_{m}\right)$-action on $\mathrm{V}$, where the multiplicative group $\mathbb{G}_{m}$ acts by rescaling, with weight denoted $D$. Our base ring is thus the larger polynomial ring $A_{T \times \mathbb{G}_{m}}^{*}(p t)=\operatorname{Sym}\left(T^{*}\right)[D]$, and all the weights $\{D+\lambda, \lambda \in$ $\left.\mathrm{T}^{*}\right\}$ live in an open half-space, making it easy to define Fourier transforms.

Proposition 2.2. Let $\mathrm{X} \subseteq \mathbb{P V}$ be a projective scheme invariant under the action of a torus $\mathrm{T}$ on $\mathrm{V}$. Let $\widehat{\mathrm{X}} \subseteq \mathrm{V}$ be the affine cone over $\mathrm{X}$, considered as a $\left(\mathrm{T} \times \mathbb{G}_{\mathrm{m}}\right)$ space.

Let $e_{\overrightarrow{0}} \widehat{X}:=[\widehat{X}] /[\overrightarrow{0}]$ be the equivariant multiplicity [Ro89] of $\widehat{X}($ at $\overrightarrow{0})$, where $[\widehat{X}],[\overrightarrow{0}] \in A_{T \times \mathbb{G}_{m}}^{*}(V)$ denote the $\left(T \times \mathbb{G}_{m}\right)$-equivariant classes, and $e_{\overrightarrow{0}} \widehat{X}$ lives in the ring of fractions. (The denominator $[\overrightarrow{0}]$ is the product of the weights of $\mathrm{T} \times \mathbb{G}_{\mathrm{m}}$ on $\mathrm{V}$.)

Assume now for convenience that $\mathrm{T}$ acts locally freely on $\mathrm{X}$. (We can achieve this by breaking $\mathrm{X}$ into components, and quotienting $\mathrm{T}$ by the kernel of the action.) Let $\mathrm{f}_{\mathrm{X}}: \mathrm{t}^{*} \times \mathbb{R} \rightarrow \mathbb{R}$ be the piecewise-homogeneous-polynomial function such that

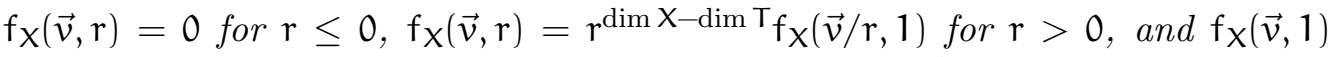
is the Duistermaat-Heckman function. Then $\mathrm{f}_{\mathbf{X}}$ and $e_{\overrightarrow{0}} \widehat{X}$ are related by Fourier transform. 
Proof. This is an easy version of [Ro89, theorem 2.1], though that is stated for the more difficult complex-analytic case.

One cheap proof in our algebro-geometric setting here is to note that both $[\widehat{X}]$ and $\mathrm{DH}(\mathrm{X}, \mathrm{T})$ are constant in locally free T-equivariant families, such as provided by Gröbner degenerations to monomial schemes, and both behave additively under the decomposition of $X$ into its top-dimensional components $X_{i}$ with multiplicities $m_{i}$. The components of monomial schemes are T-invariant linear subspaces. We are thus reduced to checking the easy case that $\widehat{X} \leq \mathrm{V}$ is a $\mathrm{T}$ invariant linear subspace; both sides become $1 / \Pi(D+\lambda)$ where $\lambda$ runs (with multiplicity) over the the T-weights in the vector space $\widehat{X}$.

In section 4.2, we will use the following proposition to constrain the coefficients $\left\{v_{\gamma}\right\}$.

Proposition 2.3. Continue the notation of proposition 2.2.

Let $\mathrm{f} \in \mathrm{X}^{\top}$, and assume that the $\Phi_{\mathrm{T}}(\mathrm{f})$-weight space $\mathrm{L}$ in $\mathrm{V}$ is one-dimensional (i.e. $\mathbb{P L}=\mathrm{f}$ ). Let $\mathrm{C}_{\mathrm{f}} \mathrm{X} \subseteq \mathrm{T}_{\mathrm{f}} \mathbb{P} \mathrm{V}$ denote the normal cone at $\mathrm{f}$ in $\mathrm{Y}$ and the tangent space at $\mathrm{f}$ to $\mathbb{P V}$, respectively. The ( $\mathrm{T}$-invariant) tangent cone carries a Chow class $\left[\mathrm{C}_{\mathrm{f}} \mathrm{X} \subseteq \mathrm{T}_{\mathrm{f}} \mathbb{P V}\right] \in \mathrm{A}_{\mathrm{T}}^{*}\left(\mathrm{~T}_{\mathrm{f}} \mathbb{P} \mathrm{V}\right) \cong \operatorname{Sym}\left(\mathrm{T}^{*}\right)$. Denote by $\left[\{\overrightarrow{0}\} \subseteq \mathrm{T}_{\mathrm{f}} \mathrm{X}\right] \in \mathrm{A}_{\mathrm{T}}^{*}\left(\mathrm{~T}_{\mathrm{f}} \mathrm{X}\right)$ the evident Chow class (a product of $\mathrm{T}$-weights).

Then specializing the following rational functions in $\mathrm{A}_{\mathrm{T} \times \mathbb{G}_{\mathrm{m}}}^{*}(\mathrm{pt})$ at $\mathrm{D}=-\Phi_{\mathrm{T}}(\mathrm{f})$, we have

$$
[\widehat{\mathrm{X}}] /[0 \times \mathrm{L}] \equiv\left[\mathrm{C}_{\mathrm{f}} \mathrm{X} \subseteq \mathrm{T}_{\mathrm{f}} \mathbb{P V}\right] /\left[[\overrightarrow{0}\} \subseteq \mathrm{T}_{\mathrm{f}} \mathbb{P V}\right]
$$

where neither side involves division by 0 .

Proof. Let $\mathrm{T}^{\prime} \leq \mathrm{T} \times \mathbb{G}_{\mathrm{m}}$ be the pointwise stabilizer of $\mathrm{L}$, so $\left(\mathrm{T}^{\prime}\right)^{*}$ can be naturally identified with $\left(\mathrm{T}^{*} \times \mathbb{Z} \mathrm{D}\right) /\left\langle\mathrm{D}+\Phi_{\mathrm{T}}(\mathrm{f})\right\rangle$. Let $\mathrm{H}$ be the unique T-invariant complement to $\mathrm{L}$, so $(0,1) \in \mathrm{H} \times\{1\} \subseteq \mathrm{V}$ provides a $\mathrm{T}^{\prime}$-invariant model for an open neighborhood of $f \in \mathbb{P V}$.

We can regard the flat degeneration of $\widehat{X}$ to $C_{L} \widehat{X}$ (whose relation to $C_{f} X$ we discuss in a moment) as an embedded degeneration inside $V$, as follows. Let $Q$ : $\mathbb{G}_{m} \rightarrow \mathrm{GL}(\mathrm{H} \oplus \mathrm{L})$ act by $\mathrm{Q}(z) \cdot(\mathrm{h}, \ell):=(z h, \ell)$. Then the flat $\operatorname{limit}_{\mathrm{z}} \lim _{z \rightarrow \infty} \mathrm{Q}(z) \cdot \widehat{\mathrm{X}}$ is easily identified with $C_{L} \widehat{X}$. By proposition 2.1, we get an equation

$$
[\widehat{X}]=\left[C_{L} \widehat{X}\right] \quad \in A_{T \times \mathbb{G}_{m}}^{*}(V) .
$$


We can similarly identify the flat $\operatorname{limit}_{\lim _{z \rightarrow \infty}} \mathrm{Q}(z) \cdot(\widehat{\mathrm{X}} \cap(\mathrm{H} \times\{1\}))$ with $\mathrm{C}_{(0,1)}(\widehat{\mathrm{X}} \cap$ $(\mathrm{H} \times\{1\}))$. Using the $\left(\mathrm{Q} \times \mathrm{T}^{\prime}\right)$-equivariant model $\mathrm{H} \times\{1\}$ above, this can in turn be $\mathrm{T}^{\prime}$-equivariantly identified with $\mathrm{C}_{\mathrm{f}} \mathrm{X}$.

Consider now the projection $\pi: C_{L} \widehat{X} \rightarrow L$, where each fiber $\pi^{-1}(\ell)$ is a subscheme of $\mathrm{H}$. Since $\pi$ is $\mathbb{G}_{\mathrm{m}}$-equivariant, the fibers are constant except for possibly the fiber over 0 . So $C_{L} \widehat{X}$ is supported on $\left(\pi^{-1}(1) \times L\right) \cup\left(\pi^{-1}(0) \times 0\right)$. Therefore

$$
\left[C_{\mathrm{L}} \widehat{X}\right]=\left[\pi^{-1}(1) \times \mathrm{L}\right]+\left(\mathrm{D}+\Phi_{\mathrm{T}}(\mathrm{f})\right) \mathrm{q}
$$

for some polynomial $q \in A_{T \times \mathbb{G}_{m}}^{*}(H)$, where the factor $D+\Phi_{T}(f)$ comes from $[0 \in$ L]. (This $\mathrm{q}$ is not necessarily the class of $\pi^{-1}(0)$, but a sum over its components, with some multiplicities we will not determine.) Since $\mathrm{H} \oplus \mathrm{L} \rightarrow \mathrm{L}$ is Q-invariant, $\pi^{-1}(1) \cong \mathrm{C}_{(0,1)}(\widehat{\mathrm{X}} \cap(\mathrm{H} \times\{1\})) \cong \mathrm{C}_{\mathrm{f}} \mathrm{X}$. Chaining these together, and working modulo $\mathrm{D}+\Phi_{\mathrm{T}}(\mathrm{f})$, we get

$$
[\widehat{X}] \equiv\left[C_{f} X \subseteq T_{f} X\right] \quad \bmod D+\Phi_{T}(f) .
$$

We can $T^{\prime}$-equivariantly identify $T_{f} \mathbb{P V} \cong \operatorname{Hom}(L, V / L) \cong V / L$. Dividing both sides of this last equation by the $\mathrm{T}^{\prime}$-weights in that space produces the formula we seek.

2.2. B-B decompositions. In the next few lemmas we will study B-B decompositions using $\mathrm{S}$-orbit closures.

Lemma 2.1. Let $\mathrm{b} \in \mathrm{V}^{*}$, thought of as an element of $\Gamma(\mathrm{X} ; \mathcal{O}(1))$, be an $\mathrm{S}$-weight vector of weight $\mathrm{k} \in \mathbb{Z}$. Let $\mathrm{f} \in \mathrm{X}^{\mathrm{S}}$ be a fixed point, and recall $\Phi_{\mathrm{S}}(\mathrm{f}) \in \mathbb{Z}$ denotes the weight of $\mathrm{S}$ on $\left.\mathcal{O}(1)\right|_{\mathrm{f}}$.

- If $\mathrm{k}>\Phi_{\mathrm{S}}(\mathrm{f})$, then $\mathrm{b}$ vanishes at $\mathrm{f}$.

- If $\mathrm{k}<\Phi_{\mathrm{S}}(\mathrm{f})$, then $\mathrm{b}$ vanishes on all of $\overline{\mathrm{X}}_{\mathrm{f}}$.

- If $\mathrm{k}=\Phi_{\mathrm{S}}(\mathrm{f})$, then on $\overline{\mathrm{X}}_{\mathrm{f}}, \mathrm{b}$ is unique up to scale.

- If $\mathrm{b}$ does not vanish at $\mathrm{f}\left(\right.$ so $\left.\mathrm{k}=\Phi_{\mathrm{S}}(\mathrm{f})\right)$, then $\mathrm{b}$ does not vanish on $\mathrm{X}_{\mathrm{f}}$.

Proof. We start with the case $X=\mathbb{P}^{1}, f=\overrightarrow{0}$, and therefore $X_{f}=\mathbb{P}^{1} \backslash \vec{\infty}$. Let $h$ be the order of the global stabilizer subgroup scheme $\left\{z \in \mathbb{F}^{\times}: S(z) \cdot \overrightarrow{1}=\overrightarrow{1}\right\}$. An S-equivariant line bundle $\mathcal{L}$ on $\mathbb{P}^{1}$ is classified by its degree $\mathrm{d}$, and the $\mathrm{S}$-weight on the fiber over 0 , in this case $\Phi_{S}(f)$. Then the weights in the representation $\Gamma\left(\mathbb{P}^{1} ; \mathcal{L}\right)$ are $\left(\Phi_{S}(f), \Phi_{S}(f)+h, \ldots, \Phi_{S}(f)+d h\right)$, and each weight space is 1-dimensional. 
In particular, if $k<\Phi_{\mathrm{S}}(\mathrm{f})$, the weight $k$ does not occur in this space of sections. So $b$ is the zero section. This proves the second statement ( still for $X=\mathbb{P}^{1}$ ).

For the others, note that the weight $\Phi_{S}(f)+i$ section vanishes at $f$ to order $i$. This proves the first statement, and this plus the 1-dimensionality together prove the third. For the fourth, note that the only S-covariant section that doesn't vanish at $f$ is the $i=0$ one, which vanishes only at $\vec{\infty}$, hence not on $X_{f}$. This settles $\mathrm{X}=\mathbb{P}^{1}$.

Now we consider the case of general $X$. Let $x$ be a point of $X_{f}$. Define an S-equivariant map $\mathbb{F}^{\times} \rightarrow \mathbb{P V}$ by $z \mapsto S(z) \cdot x$, and use the projectivity of $X$ to extend to an S-equivariant map $\mathbb{P}^{1} \rightarrow \mathbb{P V}$ (which takes $0 \mapsto f$ since $x \in X_{f}$ ). Pull back $\mathcal{O}(1)$ to $\mathbb{P}^{1}$ and apply the previous analysis.

Most of the published results about B-B decompositions concern the case that $\mathrm{X}$ is smooth, or at least normal, with the following as a rare exception:

Lemma 2.2. [Ko78, section 2] Define the opposite B-B decomposition $X=$ $\coprod_{f} X^{f}$ using the inverse action of $S$ on $X, S^{\prime}(z):=S\left(z^{-1}\right)$. Then for each $f \in X^{S}$, $\operatorname{dim} X^{f}+\operatorname{dim} X_{f} \geq \operatorname{dim} X$.

Konarski also handles the case when $X^{S}$ is not isolated, which gives an extra term we may omit. He only states the lemma (as a corollary to theorem 3, the normal case) for the case $X$ irreducible (or at least, "a variety"), but this generalizes easily: when $X=\bigcup_{i} X(i)$ is the decomposition into irreducible components then $X_{f}=\bigcup X(i)_{f}, X^{f}=\bigcup X(i)^{f}$, so

$$
\begin{aligned}
\operatorname{dim} X^{f} & +\operatorname{dim} X_{f}=\max _{i} \operatorname{dim} X(i)^{f}+\max _{j} \operatorname{dim} X(j)_{f} \\
& \geq \max _{i}\left(\operatorname{dim} X(i)^{f}+\operatorname{dim} X(i)_{f}\right) \geq \max _{i} \operatorname{dim} X(i) \quad \text { the irreducible case } \\
& =\operatorname{dim} X .
\end{aligned}
$$

To see a (normal) example where the inequality is strict, tilt a square pyramid $\mathrm{P}$ up on one edge, and let $\mathrm{f}$ be the apex, with $\overline{\mathrm{P}_{\mathrm{f}}}, \overline{\mathrm{P}^{f}}$ both being triangles. Then the inequality is $2+2>3$.

Say that $X$ has a unique supporting fixed point if $X=\overline{X_{f}}$ for some $f \in X^{\top}$. This will be part of a more general definition in the next section, but is an 
important enough special case that we introduce the notation $\min (X)=f$ for it. If $X \neq \overline{X_{f}}$ for any $f \in X^{\top}$, then $\min (X)$ is undefined.

Most authors using B-B decompositions remark somewhere that if $\mathrm{X}$ is irreducible, it has a unique supporting fixed point, called the sink. (Proof: exactly one $B-B$ stratum $X_{f}$ is open, and $X$ is the closure of that $X_{f}$.) Irreducibility is an unnatural condition for us, as any nonempty $\overline{X_{f_{0}, \ldots, f_{i}}}$ also has a unique supporting fixed point, $f_{i}$, though it may be reducible even when $X$ itself is irreducible (see the tilted octahedron example in section 1.2.2).

Corollary 2.1. Let $\mathrm{X}$ have a unique supporting fixed point, and let $\mathrm{f} \in \mathrm{X}^{\mathrm{S}}, \mathrm{f} \neq$ $\min (X)$.

Then there exists a map $\beta: \mathbb{P}^{1} \rightarrow \mathrm{X}, \mathrm{S}$-equivariant with respect to the standard action of $\mathbb{G}_{m}$ on $\mathbb{P}^{1}$, such that $\beta(\infty)=f \neq \beta(0)$. Moreover

$$
\Phi_{S}(\beta(\infty))-\Phi_{S}(\beta(0))=\operatorname{deg} \beta \cdot \operatorname{deg} \beta\left(\mathbb{P}^{1}\right)
$$

meaning the degree of the map $\beta$ to its image, times the projective degree of its image curve.

In particular each $\mathrm{f} \neq \min (\mathrm{X})$ has $\Phi_{\mathrm{S}}(\mathrm{f})>\Phi_{\mathrm{S}}(\min (\mathrm{X}))$.

Proof. The assumption on $X$ says $X=\overline{X_{\min (X)}}$, and the assumption on $f$ says $X_{f} \subseteq \overline{X_{\min (X)}} \backslash X_{\min (X)}$. Hence $\operatorname{dim} X_{f}<\operatorname{dim} X$. By lemma 2.2, $\operatorname{dim} X^{f}>0$, so there exists a point $X \in X^{f} \backslash\{f\}$, automatically not $S$-invariant. Define the map $\beta: \mathbb{P}^{1} \rightarrow \overline{X^{f}}$ by extending

$$
\beta: z \mapsto S(z) \cdot x, \quad z \in \mathbb{G}_{m}
$$

so $\beta(\infty)=f$ by choice of $x$. Then by the same analysis as in lemma 2.1 (and with the same notation $h, d), \Phi_{S}(\beta(\infty))-\Phi_{S}(\beta(0))=h d$.

This shows that for each $f \neq \min (X)$, there exists some other $g \in X^{S}$ (namely $g=\beta(0))$ such that $\Phi_{S}(f)>\Phi_{S}(g)$. By induction on the finite set $\Phi_{S}\left(X^{\top}\right)$, for each $f \neq \min (X)$ we have $\Phi_{S}(f)>\Phi_{S}(\min (X))$.

The assumption of projectivity is very clearly necessary here, since otherwise $X$ could be $\mathbb{P}^{1}$ with 0 and $\infty$ identified. (The reason that $X$ is sometimes assumed to be normal, as in much of [Ko78], is to ensure that T-invariant affine open sets on it possess closed equivariant affine embeddings.) 
Lemma 2.3. Assume $\mathrm{X}$ has a unique supporting fixed point $\min (\mathrm{X})$. Let $\mathrm{W} \leq \mathrm{V}$ be the smallest linear subspace containing $\widehat{\mathrm{X}}$. Then the $\Phi_{\mathrm{T}}(\min (\mathrm{X}))$-weight space in $\mathrm{W}$ is 1-dimensional.

Proof. Obviously we may shrink $\mathrm{V}$ to $\mathrm{W}$ from the outset.

To see that the weight space is nonzero, consider $h \in \mathrm{V}^{*}$ as an element of $\Gamma(X ; \mathcal{O}(1))$, and choose an $h$ that does not vanish at $\min (\mathrm{X})$. (We know such an $\mathrm{h}$ exists because $X$ is projectively embedded, rather than merely carrying an ample line bundle.) Expand $h$ as a sum of T-weight vectors; at least one of them must not vanish at $\min (X)$, and let $b$ be that term. Note that we can determine the $\mathrm{T}$-weight of $\mathrm{b}$ - it must be the $\mathrm{T}$-weight on the line $\left.\mathcal{O}(1)\right|_{f}$.

By assumption $X=\overline{X_{\min (X)}}$. Then the last conclusion of lemma 2.1 gives us the uniqueness of $b$ up to scale.

An even smaller $W$ will be used in proposition 2.4.

Results like the following are often attributed to [He81] (at least for $\mathbf{X}$ smooth irreducible), but I was not able to locate an exact reference therein. The last part is quite close to [BB76, theorem 3] (again, only stated for the smooth case, though his proof generalizes).

Lemma 2.4. Let $\mathrm{X} \subseteq \mathbb{P V}, \mathrm{T}, \mathrm{S}$ be as in theorem 1. Assume $\mathrm{X}$ has a unique supporting fixed point. Then there is a $\mathrm{T}$-invariant hyperplane $\mathbb{P H}$ in $\mathbb{P V}$ not containing $\min (\mathrm{X})$, and the subscheme $\mathbb{P H} \cap \mathrm{X}$ does not depend on the choice of $\mathbb{P H}$. As a set, $\mathbb{P H} \cap \mathrm{X}=\bigcup_{\mathrm{f} \neq \min (\mathrm{X})} \mathrm{X}_{\mathrm{f}}$.

Proof. Existence of the desired $\mathbb{P H}$, or equivalently, of a $\mathrm{T}$-weight vector $\mathrm{b}$ not vanishing at $\min (\mathrm{X})$, is given by lemma 2.3 , which also gives the uniqueness of $\mathbb{P H} \cap \mathrm{X}$.

By corollary 2.1, $\Phi_{S}(f)>\Phi_{S}(\min (X))$ for each $f \neq \min (X)$. Then by lemma $2.1, \mathrm{~b}$ vanishes on $\overline{X_{f}}$, and doesn't vanish on $X_{\min (X)}$. Hence $\mathbb{P H} \cap X=\bigcup_{f \neq \min (X)} X_{f}$ as a set.

It is really in this lemma that the assumption of isolated fixed points becomes crucial. Thanks to this lemma, to cut down from $X$ to the union of smaller B-B strata (or a scheme supported thereon) it suffices to take a hyperplane section, which by proposition 2.2 will let us inductively compute equivariant Chow classes. 
The following result is, in some sense, a tightest possible version of lemma 2.3. Essentially the same idea was used in [Br98, proofs of theorem 17 and corollary 19]. We won't need it for the proofs of the main theorems, but it will appear in section 4.2 .

Proposition 2.4. Let $\mathrm{W} \leq \mathrm{V}$ be the linear span of the points $\mathrm{X}^{\top}$, and pick a $\mathrm{T}$-equivariant projection $\hat{\beta}: \mathrm{V} \rightarrow \mathrm{W}$. Then the induced map $\beta: \mathrm{X} \rightarrow \mathbb{P W}$ is well-defined (has no basepoints), finite, and T-equivariant.

Proof. If $\vec{v} \in \widehat{X} \backslash \overrightarrow{0}$, then $\mathbb{P} \vec{v} \in X_{f}$ for some $f \in X^{\top}$. Let $K:=\operatorname{ker} \hat{\beta}$. Since the line over $f$ is not contained in $K$, there is an element of $K^{\perp} \leq V^{*}$ not vanishing at $f$, and hence (as explained in the proof of lemma 2.3) a T-weight vector $b_{f} \in K^{\perp}$ not vanishing at $f$. By lemma 2.1 the function $b_{f}$ doesn't vanish on $X_{f}$.

Hence the subscheme $\left\{\vec{v} \in \widehat{X}:\langle b, \vec{v}\rangle=0 \forall b \in K^{\perp}\right\}$ is supported at the origin (and therefore of finite length), which shows the lack of basepoints. Since the map $\widehat{X} \rightarrow W$ is dilation-equivariant, the fiber over $\overrightarrow{0}$ is the largest fiber, which shows the finiteness of the map. The T-equivariance is clear.

When $X$ is reduced, the map $\beta: X \rightarrow \mathbb{P W}$ is termed a branchvariety of $\mathbb{P W}$ in $[\mathrm{AK}]$, where we studied families of such maps. This will also be the point of view in $[\mathrm{Kn}]$.

Theorem 4 below will be a formula for the equivariant multiplicity $[\widehat{X}] /[\overrightarrow{0}]$, with a surprisingly small actual denominator. As in [Br98, proofs of theorem 17 and corollary 19], one can use proposition 2.4 to predict already that the denominator divides $\prod_{f \in X^{\top}}\left(D+\Phi_{T}(f)\right)$, though to carry this out would involve introducing some definitions (e.g. the D-H measures of modules and cycles) we do not take space for here.

\section{Proofs of the main theorems}

Throughout section 3.1 we work with algebraic sets, rather than schemes, and do not bother to include the caveat "as a set" after each claimed equality.

\subsection{Supporting fixed points and closure chains in the B-B decompo-} sition. Call $f \in X^{S}$ a supporting fixed point if the B-B stratum $X_{f}$ contains an open set in $X$. Since the B-B decomposition is into finitely many strata, one 
of them must contain an open set, so every B-B decomposition has a supporting fixed point. In the Stanley-Reisner case $X=X(\Delta)$, the point $i$ is a supporting fixed point iff there exists a facet (meaning, a maximal face) $F \in \Delta$ with $\min (F)=i$.

When $X$ has a unique supporting fixed point (e.g. $X$ irreducible), $X_{\min } X$ is actually open in $X$, rather than merely containing an open set. But more generally this can fail: for an example let $X=\operatorname{Proj} \mathbb{C}\left[x_{1}, x_{2}, x_{3}\right] /\left\langle x_{1} x_{3}\right\rangle$ be the StanleyReisner scheme of a union of two intervals, and $f=2$. (Perhaps the term "sink" should be reserved for those $f$ with open $X_{f}$.) If we had required this more restrictive condition in the definition of supporting fixed point, we wouldn't have lemmas 3.1 or 3.3 .

Lemma 3.1. Let $\mathrm{F} \subseteq \mathrm{X}^{\top}$ be the set of supporting fixed points. Then $\mathrm{X}=$ $\overline{\bigcup_{f \in F} X_{f}}=\bigcup_{f \in F} \overline{X_{f}}$.

In particular, if $\mathrm{X}$ has only one supporting fixed point $\min (\mathrm{X})$, then $\mathrm{X}=\overline{\mathrm{X}_{\min }(\mathrm{X})}$ (matching the terminology from section 2.2).

Proof. The proof is pure point-set topology. If $\overline{X \backslash Y} \subsetneq X$, then $Y$ contains the nonempty open set $X \backslash \overline{X \backslash Y}$. Contrapositively, if $Y_{1}=Y$ doesn't contain an open set in $X$, then $X \backslash Y_{1}$ is dense in $X$.

If $Y_{2} \subseteq X, Y_{2} \cap Y_{1}=\emptyset$ also contains no open set in $X$, then it contains no open set in $X \backslash Y_{1}$, so $X \backslash\left(Y_{1} \cup Y_{2}\right)$ is dense in $X \backslash Y_{1}$, hence dense in $X$.

Repeating this, we can remove finitely many subsets that each contain no open set in $X$, with the remainder still dense in $X$. Hence $X \backslash \bigcup_{f \notin F} X_{f}$ is dense in $X$. By the B-B decomposition, this subset is $\bigcup_{f \in F} X_{f}$.

Finally, $X \supseteq \bigcup_{f \in F} \overline{X_{f}} \supseteq \overline{\cup_{f \in F} X_{f}}=X$, hence all three are equal.

Lemma 3.2. Let $\mathrm{Y} \subseteq \mathrm{X}$ be closed and $\mathrm{S}$-invariant, e.g. if $\mathrm{Y}$ is an irreducible component of $\mathrm{X}$. Then $\mathrm{Y}$ has a B-B decomposition, with $\mathrm{Y}_{\mathrm{f}}=\mathrm{Y} \cap \mathrm{X}_{\mathrm{f}}$ for $\mathrm{f} \in \mathrm{Y}^{\mathrm{S}}$ (as noted in [BB76]). Each closure chain $\gamma$ for $\mathrm{Y}$ is a closure chain for $\mathrm{X}$, with $\gamma \subseteq \mathrm{Y}^{S}$. 
Proof. Since $\mathbb{G}_{m}$ is connected, its action on the set of components of $\mathrm{X}$ is trivial, which is why irreducible components are S-invariant. The next claim is tautological:

$Y_{f}=\left\{y \in Y: \lim _{z \rightarrow 0} S(z) \cdot y=f\right\}=\left\{y \in X: y \in Y, \lim _{z \rightarrow 0} S(z) \cdot y=f\right\}=Y \cap X_{f}$.

Obviously the closure chains $\gamma$ for $Y$ have $\gamma \subseteq Y^{S}$, and $\overline{Y_{\gamma}} \subseteq \overline{X_{\gamma}}$; thus each closure chain for $\mathrm{Y}$ is a closure chain for $\mathrm{X}$.

The converse is not true: it is often the case that $\gamma \subseteq Y^{S}$ is not a closure chain for $Y \subseteq X$ even though it is a closure chain for $X$, and this can happen even when $\mathrm{Y}$ is irreducible (consider $\mathrm{Y}=\mathrm{F}_{1}$ as in section 1.2.2, with $\mathrm{X}=\mathrm{Y} \cup \mathbb{P}^{1}$ intersecting at the points $a, c)$. Our best partial converse will be corollary 3.1 below.

Lemma 3.3. If $\mathrm{Y} \subseteq \mathrm{X}$ is an irreducible component, then its unique supporting fixed point $\min (\mathrm{Y})$ is also a supporting fixed point of $\mathrm{X}$. In particular, every irreducible component of $\overline{\mathrm{X}_{\mathrm{f}}}$ contains $\mathrm{f}$.

Proof. If $\mathrm{Y}$ is a component, it contains an open set $\mathrm{Y}^{\circ}$ in $\mathrm{X}$, so it must meet some $X_{f}$ for $f$ a supporting fixed point, and we may pick $y \in Y^{\circ} \cap X_{f}$. Since $Y$ is closed and S-invariant, $\lim _{z \rightarrow 0} S(z) \cdot y=f$ lies in $Y$.

Since $Y^{\circ} \cap X_{f}$ contains a nonempty open set in $Y$ (irreducible), it is dense. It is contained in $\mathrm{Y} \cap \mathrm{X}_{\mathrm{f}}$, which by lemma 3.2 is $\mathrm{Y}_{\mathrm{f}}$, and this makes $\mathrm{f}$ a supporting fixed point of $Y$. Since $Y$ is irreducible, it is the unique such.

The second half of the following lemma is very similar to one in [BB76], where it is only proven under the assumption that each intersection $X_{f} \cap X^{g}$ is transverse.

Lemma 3.4. Assume $\mathrm{X}$ has a unique supporting fixed point, and let $\mathrm{f} \in \mathrm{X}^{\mathrm{S}}$, $\mathrm{f} \neq \min (\mathrm{X})$. Then $\operatorname{dim} \overline{X_{\mathrm{f}}}<\operatorname{dim} \mathrm{X}$. Consequently, any closure chain for $\mathrm{X}$ has at most $1+\operatorname{dim} \mathrm{X}$ elements.

Proof. This can be proven using lemma 2.4, or even more directly, as we do now.

$$
X_{f} \subseteq X \backslash X_{\min (X)}=\overline{X_{\min (X)}} \backslash X_{\min (X)}
$$

and the right-hand side has lower dimension than $X_{\min (X)}$. Then $\operatorname{dim} \overline{X_{f}}=$ $\operatorname{dim} X_{f}<\operatorname{dim} X_{\min (X)}$. 
Now let $\left(f_{0}, \ldots, f_{m}\right)$ be a closure chain for $X$. Then

$$
\overline{X_{f_{0}}} \supsetneq \overline{X_{f_{0}, f_{1}}} \supsetneq \ldots \supsetneq \overline{X_{f_{0}, \ldots, f_{m}}}
$$

where by its construction, $\overline{X_{f_{0}, \ldots, f_{i}}}$ has a unique supporting fixed point $f_{i}$. Hence by the above, the dimensions of these spaces are strictly decreasing in this chain, and there must therefore be at most $1+\operatorname{dim} X$ of them.

To define the coefficients $v_{\gamma}$ of theorem 1 , we will need a refinement of the notion of closure chain, which we develop in a series of lemmas.

Lemma 3.5. Let $\bar{\gamma}=\left(\left(f_{0} \in Y_{0}\right),\left(f_{1} \in Y_{1}\right), \ldots,\left(f_{m} \in Y_{m}\right)\right)$ be a list of pairs $\left(f_{i} \in X^{\top}, Y_{i} \subseteq X\right)$ such that each $Y_{i \geq 0}$ is an irreducible component of $\overline{\left(Y_{i-1}\right)_{f_{i}}}$, interpreting $\mathrm{Y}_{-1}$ as $\mathrm{X}$. Assume also that $\gamma=\left(\mathrm{f}_{0}, \ldots, \mathrm{f}_{\mathrm{m}}\right)$ is nonrepeating.

Then $\gamma=\left(f_{0}, \ldots, f_{m}\right)$ is a closure chain, and we call $\bar{\gamma}$ a witness to $\gamma$ in $X$.

If $\mathrm{Y} \subseteq \mathrm{X}$ is a component, then any witness to a closure chain in $\mathrm{Y}$ is a witness to the same closure chain in $\mathrm{X}$. Conversely, any witness $\bar{\gamma}=\left(\left(\mathrm{f}_{\mathrm{O}} \in \mathrm{Y}_{\mathrm{O}}\right), \ldots,\left(\mathrm{f}_{\mathrm{m}} \in \mathrm{Y}_{\mathrm{m}}\right)\right)$ to a closure chain in $\mathrm{X}$ is a witness in $\mathrm{Y}$ too, if $\mathrm{Y} \supseteq \mathrm{Y}_{0}$.

Proof. We need to show that $\overline{X_{f_{0}, \ldots, f_{m}}} \neq \emptyset$. So we show inductively that each $\mathrm{Y}_{\mathrm{k}} \subseteq \overline{\mathrm{X}_{\mathrm{f}_{0}, \ldots, \mathrm{f}_{\mathrm{k}}}}$. First,

$$
\mathrm{Y}_{\mathrm{o}}=\overline{\left(\mathrm{Y}_{\mathrm{O}}\right)_{\mathrm{f}_{\mathrm{o}}}}=\overline{\left(\mathrm{Y}_{\mathrm{O}}\right) \cap \mathrm{X}_{\mathrm{f}_{\mathrm{o}}}} \subseteq \overline{\mathrm{X}_{\mathrm{f}_{0}}} \quad \text { using lemma 3.2. }
$$

Then for $i>0$, using lemma 3.2 and induction,

$$
Y_{i} \subseteq \overline{\left(Y_{i-1}\right)_{f_{i}}}=\overline{Y_{i-1} \cap X_{f_{i}}} \subseteq \overline{\overline{X_{f_{0}, \ldots, f_{i-1}}} \cap X_{f_{i}}}=\overline{X_{f_{0}, \ldots, f_{i}}} .
$$

So $\overline{X_{f_{0}, \ldots, f_{m}}} \supseteq Y_{m}$ and hence is nonempty, making $\gamma$ a closure chain.

Note that the sequence $\left(Y_{i}\right)$ is weakly decreasing, since $Y_{i} \subseteq \overline{\left(Y_{i-1}\right)_{f_{i}}} \subseteq \overline{Y_{i-1}}=$ $Y_{i-1}$. Hence the conditions $Y_{i} \subseteq X$ are equivalent to $Y_{0} \subseteq X$. The final condition is that $Y_{0}$ is a component of $\overline{X_{f_{0}}}$.

The second and third claims are then tautological, as the definition of witness only involves the ambient space in the condition $Y_{0} \subseteq X$.

We make two remarks about the definition. The $\left(f_{i}\right)$ in a witness can be recovered from the $\left(Y_{i}\right)$ as $f_{i}=\min \left(Y_{i}\right)$, but it seems unnatural to leave the $\left(f_{i}\right)$ out of the definition as it doesn't simplify the axioms on the $\left(Y_{i}\right)$. Also, one could formulate a weaker notion of witness, a chain of varieties in which each $Y_{i}$ is 
S-invariant and irreducible with $\min \left(Y_{i}\right)=f_{i}$, just not necessarily a component. But the following lemma suggests that we will not need this greater generality.

Lemma 3.6. Every closure chain $\gamma=\left(f_{0}, \ldots, f_{m}\right)$ has witnesses, and only finitely many thereof.

Proof. The proof of existence is by induction on $m$. If $m=0$ then this is easy: pick some component $Y_{0}$ of $\overline{X_{f_{0}}}$, and apply lemma 3.3 to know that $f_{0}=\min \left(Y_{0}\right)$.

Now assume $m>0$. Tautologically, $\left(f_{1}, \ldots, f_{m}\right)$ is a closure chain of $\overline{X_{f_{0}, f_{1}}}$, and so has a witness $\left(\left(f_{1} \in Y_{1}\right), \ldots,\left(f_{m} \in Y_{m}\right)\right)$ by induction. Since $Y_{1}$ is irreducible, we may choose an irreducible component $Y_{0}$ of $\overline{X_{f_{0}}}$ containing it. By lemma 3.3, $f_{0}=\min \left(Y_{0}\right)$.

It remains to show that $Y_{1} \subseteq \overline{\left(Y_{0}\right)_{f_{1}}}$. (It will automatically be a component, since it is a component of the larger $\overline{X_{f_{0}, f_{1}}}$. Since $f_{1}=\min \left(Y_{1}\right)$, it is enough to show $\left(Y_{1}\right)_{f_{1}} \subseteq\left(Y_{0}\right)_{f_{1}}=Y_{0} \cap X_{f_{1}}$ (by lemma 3.2), and indeed we know that $\mathrm{Y}_{1} \subseteq \mathrm{Y}_{0}$ and $\left(\mathrm{Y}_{1}\right)_{\mathrm{f}_{1}} \subseteq \mathrm{X}_{\mathrm{f}_{1}}$.

If one imagines enumerating witnesses to a given closure chain by picking $Y_{0}$, then $Y_{1}$, etc., then at each stage one picks an irreducible component of a projective scheme, which means finitely many choices. (Sometimes the scheme is empty and there are zero choices, if one has made a bad choice along the way; this is why we didn't use this argument to show existence.)

Corollary 3.1. If $X=\bigcup_{i} X_{i}$ is the decomposition into irreducible components, then $\Delta(X, S)=\bigcup_{i} \Delta\left(X_{i}, S\right)$.

Proof. For each closure chain $\gamma \in \Delta(X, S)$, pick a witness $\bar{\gamma}$, and an irreducible component $X_{i}$ of $X$ containing the $Y_{0}$ from $\bar{\gamma}$.

Since $\operatorname{DH}(X, T)=\sum X_{i} \operatorname{DH}\left(X_{i}, T\right)$ (for $\left\{X_{i}\right\}$ the top-dimensional primary components), and theorem 1 gives each side of this equation as a sum over topdimensional faces of the corresponding complexes, one might expect this union of the complexes to be disjoint on the top-dimensional faces. It need not be, as the second example in section 1.2.4 shows. In [Kn], it will indeed be a disjoint union of some simplicial posets that refine the simplicial complexes presented here.

Our main interest in witnesses is in the case $m=\operatorname{dim} X$, as these are the only $\gamma$ that contribute in the formula in theorem 1. 
Lemma 3.7. Let $\bar{\gamma}=\left(\left(\mathrm{f}_{0} \in \mathrm{Y}_{0}\right), \ldots,\left(\mathrm{f}_{\operatorname{dim} \mathrm{X}} \in \mathrm{Y}_{\operatorname{dim} \mathrm{X}}\right)\right)$ be a witness. Then $\mathrm{Y}_{0}$ is a top-dimensional component of $\mathrm{X}$, each $\mathrm{Y}_{\mathrm{i}+1}$ is a Weil divisor in $\mathrm{Y}_{\mathfrak{i}}$, and $\mathrm{Y}_{\operatorname{dim} \mathrm{X}}$ is the singleton $\left\{\mathrm{f}_{\operatorname{dim} \mathrm{X}}\right\}$.

Proof. By lemma $3.4 \operatorname{dim} Y_{i}+1 \leq \operatorname{dim} Y_{i-1}$, so $\operatorname{dim} Y_{i}+i \leq \operatorname{dim} Y_{0}$. Hence

$$
\operatorname{dim} X \leq \operatorname{dim} Y_{\operatorname{dim}} X+\operatorname{dim} X \leq \operatorname{dim} Y_{i}+i \leq \operatorname{dim} Y_{0}+0 \leq \operatorname{dim} X,
$$

making each one an equality: $\operatorname{dim} Y_{i}=\operatorname{dim} X-i$.

In particular, $Y_{\operatorname{dim} X}$ is 0 -dimensional. It is also irreducible, and contains $f_{\operatorname{dim} X}$.

As mentioned earlier, for purposes of computing the D-H measure of $\mathrm{X}$ we may assume $X$ is equidimensional. Under that assumption, we now show (though we won't make use of it) that maximal closure chains are maximum, i.e. have $1+\operatorname{dim} X$ elements.

Proposition 3.1. If $\mathrm{X}$ is equidimensional, then so is $\Delta(\mathrm{X}, \mathrm{S})$ (the first as a reduced scheme, the second as a simplicial complex).

In particular, if the $B-B$ decomposition is a stratification, then the $\operatorname{poset}\left(\mathrm{X}^{\top}, \geq\right)$ is a ranked poset, with the ranking given by $\mathrm{f} \mapsto \operatorname{dim} \mathrm{X}-\operatorname{dim} \mathrm{X}_{\mathrm{f}}$.

Proof. Let $\gamma$ be a maximal closure chain, and pick a witness

$$
\bar{\gamma}=\left(\left(f_{0} \in Y_{0}\right), \ldots,\left(f_{m} \in Y_{m}\right)\right)
$$

to $\gamma$ using lemma 3.6. We wish to show $m=\operatorname{dim} X$.

We claim $Y_{0}$ must be a component of $X$. For otherwise, we could pick a component $Y \subseteq X$ properly containing it, and stick $\min (Y)$ at the beginning of $\gamma$, contradicting $\gamma$ 's maximality. By $X$ 's equidimensionality, $\operatorname{dim} Y_{0}=\operatorname{dim} X$.

As in the proof of lemma 3.4, $\operatorname{dim} Y_{i} \leq \operatorname{dim} X-i$ for each $i$. We claim now that this is an equality. Otherwise, let $i$ be the least such that the inequality is strict; by the previous paragraph we know $i>0$. So $\operatorname{dim} Y_{i}<\operatorname{dim} Y_{i-1}-1$. By lemma 2.4, $Y_{i}$ is contained inside the hyperplane section $Y_{i-1} \backslash\left(Y_{i-1}\right)_{f_{i-1}}$. So we can pick a component $Z$ of $Y_{i-1} \backslash\left(Y_{i-1}\right)_{f_{i-1}}$ containing $Y_{i}$. Since $Y_{i-1} \backslash$ $\left(Y_{i-1}\right)_{f_{i-1}}$ is pure of codimension 1 inside $Y_{i-1}, Z$ properly contains $Y_{i-1}$. Now $\left(\left(f_{0} \in Y_{0}\right), \ldots,\left(f_{i-1} \in Y_{i-1}\right),(\min (Z) \in Z),\left(f_{i} \in Y_{i}\right), \ldots,\left(f_{m} \in Y_{m}\right)\right)$ is a witness, so by interposing $\min (Z)$ we have extended $\gamma$, contradiction. 
Finally, we claim $Y_{m}$ is a point. Otherwise, the hyperplane section $Y_{m} \backslash\left(Y_{m}\right)_{f_{m}}$ is nonempty, so we can pick a component $Y_{m+1}$ of it and extend $\gamma$ at the end, contradiction.

Hence $m=\operatorname{dim} X-\operatorname{dim} Y_{m}=\operatorname{dim} X-0$, as was to be shown.

This proposition is another sign of the minimality of $\Delta(X, S)$, in the following sense. Theorem 1 only makes use of the top-dimensional faces of $\Delta(X, S)$. Proposition 3.1 says (in the case that one has thrown out the lower-dimensional components) that $\Delta(\mathrm{X}, \mathrm{S})$ only has those faces implied by those top-dimensional ones, with no extraneous maximal-but-not-maximum faces.

In $[\mathrm{Kn}]$ we will give a degeneration-based proof of proposition 3.1, which will enable us to prove the following additional result: if $X$ is equidimensional and connected in codimension one (e.g. if $X$ is irreducible), then so too is $\Delta(X, S)$.

We made special mention in proposition 3.1 of the stratification case, as one can use this to show the known but perhaps surprising fact that the poset of K-orbits on a flag manifold $G / B$, for $K$ a symmetric subgroup of $G$, is a ranked poset. This poset is also that of the B-orbits on $\mathrm{G} / \mathrm{K}$, which is an order ideal in the poset of B-orbits on the wonderful compactification of G/K [DCP73]. Those orbits are given by a B-B decomposition, and this proposition then provides the proof.

3.2. The main theorems. We first define a refinement of the $\left\{v_{\gamma}\right\}$, using the witnesses $\bar{\gamma}=\left(\left(f_{0} \in Y_{0}\right),\left(f_{1} \in Y_{1}\right), \ldots,\left(f_{\operatorname{dim} x} \in Y_{\operatorname{dim} X}\right)\right)$ to $\gamma$. Hereafter in this section, $\bar{\gamma}$ will denote a witness $\left(\left(f_{0} \in Y_{0}\right),\left(f_{1} \in Y_{1}\right), \ldots,\left(f_{k} \in Y_{k}\right)\right)$ with the condition $\operatorname{dim} Y_{i}=\operatorname{dim} X-i$ for $i=0, \ldots, k$, though not until later will we assume $k=\operatorname{dim} X$.

Let $m_{\bar{\gamma}, 0}$ denote the multiplicity of $Y_{0}$ as a component of $X$. For each $i>0$, use lemma 2.4 to choose some $T$-invariant $\mathbb{P H}$ that misses $f_{i-1}$, with which to (well-) define the subscheme $Y_{i-1} \cap \mathbb{P H}$. This hyperplane section is equidimensional of dimension $\operatorname{dim} Y_{i-1}-1=\operatorname{dim} Y_{i}$, and we can let $m_{\bar{\gamma}, i}$ denote the multiplicity of $Y_{i}$ as a component of it. (In a moment we will prove that it is indeed a component, i.e. that $m_{\bar{\gamma}, i}>0$.) Then define

$$
v(\mathrm{X})_{\bar{\gamma}}:=\prod_{i=0}^{k} m_{\bar{\gamma}, i} \quad \text { and } \quad v_{\gamma=\left(f_{0}, \ldots, f_{\operatorname{dim} x}\right)}:=\sum_{\bar{\gamma}=\left\{\left(f_{0} \in Y_{0}\right), \ldots,\left(f_{\operatorname{dim} x} \in Y_{\operatorname{dim} x}\right)\right\}} v_{\bar{\gamma}}
$$


(The latter sum is a finite sum by lemma 3.6.)

Lemma 3.8. The numbers $\left\{\mathrm{m}_{\bar{\gamma}, \mathrm{i}}\right\},\left\{v(\mathrm{X})_{\bar{\gamma}}\right\}$, and $\left\{v_{\gamma}\right\}$ are all strictly positive.

Proof. Fix a witness $\bar{\gamma}$ and an $i>0$. Pick an $S$-invariant hyperplane $\mathbb{P H}$ not containing $f_{i-1}$. By lemma 2.4, $Y_{i-1} \cap \mathbb{P H} \supseteq \overline{\left(Y_{i-1}\right)_{f_{i}}}$, and that contains $Y_{i}$. As explained above, the dimensions match so $Y_{i}$ is a component of $Y_{i-1} \cap \mathbb{P H}$. This shows that $m_{\bar{\gamma}, i}>0$.

(In fact this is the principal place that we use algebraic geometry/Chow theory rather than topology/homology, where the singularities made the orientation ${ }^{5}$ issues look particularly fearsome.)

Thus each $v(\mathrm{X})_{\bar{\gamma}}$ is a product of positive integers, hence positive. By lemma 3.6, each $\gamma$ has some witness $\bar{\gamma}$, thus $v_{\gamma}$ is a nonempty sum of positive integers, hence positive.

That $m_{\bar{\gamma}, 0}$ has such a different definition from $m_{\bar{\gamma}, i>0}$ is a hint that $X$ should perhaps be required to be reduced from the beginning, as in $[\mathrm{AK}]$; it will indeed be so in $[\mathrm{Kn}]$.

We first prove an analogue of theorem 1 for equivariant Chow classes, and then give the straightforward equivalence with the stated theorem. Theorem 3 will also be a reasonably automatic consequence.

Theorem 4. Let $\mathrm{X} \subseteq \mathbb{P V}, \mathrm{T}, \mathrm{S}$ be as in theorem 1. Let $\widehat{\mathrm{X}} \subseteq \mathrm{V}$ be the $\left(\mathrm{T} \times \mathbb{G}_{\mathrm{m}}\right)$ invariant affine cone over $\mathrm{X}$, and $[\widehat{\mathrm{X}}] \in \mathrm{A}_{\mathrm{T} \times \mathbb{G}_{\mathrm{m}}}^{*}(\mathrm{~V}) \cong \operatorname{Sym}\left(\mathrm{T}^{*}\right)[\mathrm{D}]$ its equivariant Chow class. Then its equivariant multiplicity can be computed as follows:

$$
[\widehat{\mathrm{X}}] /[\overrightarrow{0}]=\sum_{\gamma} \frac{v_{\gamma}}{\prod_{\mathrm{f} \in \gamma}\left(\mathrm{D}+\Phi_{\mathrm{T}}(\mathrm{f})\right)}
$$

considered as an element of the fraction field of the polynomial ring $\operatorname{Sym}\left(\mathrm{T}^{*}\right)[\mathrm{D}]$, where $\gamma \in \Delta(\mathrm{X}, \mathrm{S})$ varies over the maximum-length closure chains, and the $v_{\gamma}$ are as defined above.

\footnotetext{
${ }^{5}$ Note added in proof: Susan Tolman has shown me a symplectic but nonalgebraic example in which the multiplicities are indeed negative.
} 
Proof. By the definition of the $v_{\gamma}$, the formula is obviously equivalent to the more refined sum over maximum-length witnesses

$$
[\widehat{X}] /[\overrightarrow{0}]=\sum_{\bar{\gamma}=\left\{\left(f_{0} \in Y_{0}\right), \ldots,\left(f_{\operatorname{dim} x} \in Y_{\operatorname{dim} x}\right)\right\}} \frac{v_{\bar{\gamma}}}{\prod_{i=0}^{n}\left(D+\Phi_{T}\left(f_{i}\right)\right)} .
$$

The interesting case is when $X$ is reduced and irreducible; as we now show, it is easy to handle the general case if granted this special one.

Let $\left\{\widehat{X}_{i}\right\}$ be the top-dimensional irreducible components of $\widehat{X}$ (similarly $X_{i}$ of $\mathrm{X})$, occurring with multiplicities $\mathrm{m}_{i}$. For each witness $\bar{\gamma}$ in $\mathrm{X}$, by lemmas 3.5 and $3.7 \bar{\gamma}$ is a witness in $X_{i}$ iff $Y_{0}=X_{i}$. Let $\nu_{\bar{\gamma}}^{i} \in \mathbb{N}$ denote the coefficient in the (assumed) formula for $\left[\widehat{X}_{i}\right]$ if $\widehat{X}_{i}=Y_{0}$, and 0 otherwise. Unwinding the definitions, we see $m_{i} v_{\bar{\gamma}}^{i}=v_{\bar{\gamma}}$ for $\widehat{X}_{i}=Y_{0}$, and is 0 otherwise; thus $\sum_{i} m_{i} v_{\bar{\gamma}}^{i}=v_{\bar{\gamma}}$. Then

$$
\begin{aligned}
{[\widehat{X}] /[\overrightarrow{0}] } & =\sum_{i} m_{i}\left[\widehat{X}_{i}\right] /[\overrightarrow{0}] \quad \text { by proposition } 2.1 \\
& =\sum_{i} m_{i} \sum_{\bar{\gamma}} \frac{v_{\bar{\gamma}}^{i}}{\prod_{i=0}^{n}\left(D+\Phi_{T}\left(f_{i}\right)\right)}=\sum_{\bar{\gamma}} \frac{\sum_{i} m_{i} v \frac{i}{\gamma}}{\prod_{i=0}^{n}\left(D+\Phi_{T}\left(f_{i}\right)\right)} \\
& =\sum_{\bar{\gamma}} \frac{v_{\bar{\gamma}}}{\prod_{i=0}^{n}\left(D+\Phi_{T}\left(f_{i}\right)\right)}
\end{aligned}
$$

as claimed. In each sum $\bar{\gamma}$ varies over witnesses in $\mathrm{X}$ having the (by lemma 3.4) maximum length, $1+\operatorname{dim} X$.

Now assume that $X$ is reduced and irreducible, and that the theorem has been proven in dimensions $<\operatorname{dim} X$ (for both irreducible and reducible). Since $X$ is irreducible, it has a unique supporting fixed point.

By lemma 2.4, there exists a T-invariant hyperplane $\mathbb{P H} \leq \mathbb{P V}$ not containing $\min (X)$, whose defining equation $b=0$ is of $\left(T \times \mathbb{G}_{m}\right)$-weight $D+\Phi_{T}(\min (X))$. Since $\mathbb{P H} \not \supset \min (X)$ and $X$ is irreducible, $\mathbb{P H}$ contains no component of $X$. So by proposition 2.1

$$
\left(\mathrm{D}+\Phi_{\mathrm{T}}(\min (\mathrm{X}))\right)[\widehat{\mathrm{X}}]=[\mathrm{H} \cap \widehat{\mathrm{X}}] .
$$

This $\mathrm{H} \cap \widehat{\mathrm{X}}$ has dimension $\operatorname{dim} \mathrm{X}-1$ (in fact it is equidimensional), so by induction its equivariant multiplicity has a formula of the form

$$
[H \cap \widehat{X}] /[\overrightarrow{0}]=\sum_{\gamma} \frac{v_{\gamma}^{\prime}}{\prod_{i=0}^{n}\left(D+\Phi_{T}\left(f_{i}\right)\right)}
$$


where $\gamma$ varies over the maximum-length closure chains of $\mathbb{P H} \cap \mathrm{X}$. (In this formula we write $v^{\prime}$ rather than $v$ because the formula is for $H \cap \widehat{X}$, not $\widehat{X}$.)

Since $\mathrm{X}$ is irreducible, by lemma $3.7 \mathrm{Y}_{0}=\mathrm{X}$ in any maximum witness in $\mathrm{X}$. Hence the maximum witnesses in $\mathbb{P H} \cap X$ and $X$ correspond 1:1 under the map

$$
\alpha:\left(\left(f_{1} \in Y_{1}\right), \ldots,\left(f_{\operatorname{dim}} x \in Y_{\operatorname{dim}} x\right)\right) \mapsto\left((\min (X) \in X),\left(f_{1} \in Y_{1}\right), \ldots,\left(f_{\operatorname{dim}} x \in Y_{\operatorname{dim}} X\right)\right)
$$

Since $X$ is reduced, its multiplicity is 1 , so $v_{\bar{\gamma}}^{\prime}=v_{\alpha(\bar{\gamma})}$. Together,

$$
\begin{aligned}
{[\widehat{\mathrm{X}}] /[\overrightarrow{0}] } & =\frac{1}{\mathrm{D}+\Phi_{\mathrm{T}}(\min (\mathrm{X}))}[\mathrm{H} \cap \widehat{\mathrm{X}}] /[\overrightarrow{0}] \\
& =\frac{1}{\mathrm{D}+\Phi_{\mathrm{T}}(\min (\mathrm{X}))} \sum_{\bar{\gamma}} \frac{v_{\bar{\gamma}}^{\prime}}{\prod_{i=1}^{n}\left(\mathrm{D}+\Phi_{\mathrm{T}}\left(\mathrm{f}_{\mathrm{i}}\right)\right)}=\sum_{\alpha(\bar{\gamma})} \frac{v_{\alpha(\bar{\gamma})}}{\prod_{i=0}^{n}\left(\mathrm{D}+\Phi_{\mathrm{T}}\left(\mathrm{f}_{\mathrm{i}}\right)\right)}
\end{aligned}
$$

where the left sum is over maximum-length witnesses for $\mathbb{P H} \cap \mathrm{X}$, and as argued above the right sum is over maximum-length witnesses for $\mathrm{X}$. By lemma 3.8, the coefficients are all positive.

Proof of theorem 1. The rational function $\prod_{\mathrm{f} \in \gamma}\left(\mathrm{D}+\Phi_{\mathrm{T}}(\mathrm{f})\right)^{-1}$ is the specialization of $\prod_{i=0}^{n} x_{i}^{-1}$ under the map $x_{i} \mapsto D+\Phi_{T}\left(f_{i}\right)$. Correspondingly, the Fourier transform of $\prod_{f \in \gamma}\left(D+\Phi_{T}(f)\right)^{-1}$ is the image of Lebesgue measure on $\mathbb{R}_{\geq 0}^{n+1}$ under the map $\left(\xi_{0}, \ldots, \xi_{n}\right) \mapsto \sum_{i} \xi_{i}\left(D+\Phi_{T}\left(f_{i}\right)\right)$, where $\gamma=\left(f_{0}, \ldots, f_{n}\right)$.

Now proposition 2.2, applied to theorem 4, gives theorem 1.

Recall that theorem 1 was stated as an existence result for a mysterious family of coefficients $\left\{v_{\gamma}\right\}$, that were then defined in theorem 2. The proof just given didn't explicitly use theorem 2's family of coefficients, but rather the $\left\{v_{\gamma}\right\}$ constructed by summing over witnesses $\left\{v_{\bar{\gamma}}\right\}$. To prove theorem 2 we will show that these two definitions of $\left\{v_{\gamma}\right\}$ agree.

Proof of theorem 2. We first show uniqueness, and thereby uncover a formula for $v(Z)_{\left(f_{0}, \ldots, f_{k}\right), Y}$ in terms of witnesses.

The $j=0$ case of the recurrence is

$$
v(Z)_{\left(f_{0}, \ldots, f_{k}\right), Y}=\sum_{Y_{0} \subseteq \overline{Z_{f_{0}}}, Y_{0} \supseteq Y} v(Z)_{\left(f_{0}\right), Y_{0}} v\left(Y_{0}\right)_{\left(f_{0}, \ldots, f_{k}\right), Y}
$$


where in this and in the sums below, $Y_{i}$ varies over the irreducible components of the space said to contain it. Then expand the last term, using the $j=1$ case:

$$
\begin{aligned}
v(Z)_{\left(f_{0}, \ldots, f_{k}\right), Y}= & \sum_{Y_{0} \subseteq \overline{Z_{f_{0}}},} v(Z)_{\left(f_{0}\right), Y_{0}} \sum_{Y_{1} \subseteq \overline{Z_{f_{0}}, f_{1}},} \sum_{Y_{1} \supseteq Y} v\left(Y_{0}\right)_{\left(f_{0}, f_{1}\right), Y_{1}} v\left(Y_{1}\right)_{\left(f_{1}, \ldots, f_{k}\right), Y} \\
= & \sum_{Y_{0} \subseteq \overline{Z_{f_{0}}}, Y_{1} \subseteq \overline{Z_{f_{0}}, f_{1}}, Y_{1} \supseteq Y} v(Z)_{\left(f_{0}\right), Y_{0}} v\left(Y_{0}\right)_{\left(f_{0}, f_{1}\right), Y_{1}} v\left(Y_{1}\right)_{\left(f_{1}, \ldots, f_{k}\right), Y .}
\end{aligned}
$$

Expanding the last term using the $j=1$ expansion $k-1$ more times, we get

$$
v(Z)_{\left(f_{0}, \ldots, f_{k}\right), Y}=\sum_{\left(Y_{0}, \ldots, Y_{k} \supseteq Y\right): Y_{i} \subseteq \overline{Z_{f_{0}}, \ldots, f_{i}}} v(Z)_{\left(f_{0}\right), Y_{0}} \prod_{i=1}^{k} v\left(Y_{i-1}\right)_{\left(f_{i-1}, f_{i}\right), Y_{i}} .
$$

Assumptions (2) and (3) of the recurrence tie these to the definitions at the beginning of section 3.2 :

$$
v(Z)_{\left(f_{0}\right), Y_{0}}=m_{\bar{\gamma}, 0}, \quad v\left(Y_{i-1}\right)_{\left(f_{i-1}, f_{i}\right), Y_{i}}=m_{\bar{\gamma}, i}
$$

and so

$$
\begin{aligned}
v(Z)_{\left(f_{0}, \ldots, f_{k}\right), Y}= & \sum_{\left(Y_{0}, \ldots, Y_{k} \supseteq Y\right): Y_{i} \subseteq \overline{Z_{f_{0}}, \ldots, f_{i}}} m_{\bar{\gamma}, 0} \prod_{i=1}^{k} m_{\bar{\gamma}, i} \\
= & \sum_{\left(Y_{0}, \ldots, Y_{k} \supseteq Y\right): Y_{i} \subseteq Z_{f_{0}, \ldots, f_{i}}} v(Z)_{\left(f_{0} \in Y_{0}, \ldots, f_{k} \in Y_{k}\right) .}
\end{aligned}
$$

In particular, if $Z=X$ and $k=\operatorname{dim} X$ so (by lemma 3.7) $Y=\left\{f_{k}\right\}$, this says $v(\mathrm{X})_{\left(\mathrm{f}_{0}, \ldots, \mathrm{f}_{\mathrm{dim} X} \mathrm{X}, \mathrm{Y}\right.}=\sum_{\bar{\gamma}} v(\mathrm{X})_{\bar{\gamma}}=: v(\mathrm{X})_{\gamma}$, as we wanted to show.

So far we have shown that the recurrence has at most one solution (even using only $j \leq 1$ ), and that solution reproduces the $\left\{v_{\gamma}\right\}$ used in the proof of theorem 1 . It remains to show that this solution - summing over all ways to lift $\left(f_{0}, \ldots, f_{k}\right)$ to a witness ending with $Y_{k}=Y$ - actually satisfies the recurrence, but this is easy: extend to a witness by first choosing $Y_{j}$, then choose the other $\left\{Y_{i}\right\}$ behind and ahead $Y_{j}$.

Proof of theorem 3. Our goal is to understand

$$
\left(\prod_{i=1}^{k} \alpha_{i}\right)[\widehat{X}]=\left(\prod_{i=1}^{k} \alpha_{i}\right) \sum_{\gamma} \frac{v_{\gamma}}{\prod_{f \in \gamma}\left(D+\Phi_{\mathrm{T}}(f)\right)}=\sum_{\gamma} v_{\gamma} \frac{\prod_{i=1}^{k} \alpha_{i}}{\prod_{f \in \gamma}\left(D+\Phi_{\mathrm{T}}(f)\right)}
$$

where the terms on the right are ready for multivariable partial fractions expansion. 
This will create many terms along the way of the form $q / \prod_{f \in Q}\left(D+\Phi_{T}(f)\right)$, whose Fourier transform is some complicated distribution supported on the cone positively spanned by $\left\{D+\Phi_{\mathrm{T}}(f): f \in Q\right\}$. By the assumption that $p$ is in general position, we can drop any such term for which that set $\left\{\mathrm{D}+\Phi_{\mathrm{T}}(\mathrm{f}): f \in \mathrm{Q}\right\}$ does not $\mathbb{Q}$-span $\mathrm{T}^{*} \oplus \mathbb{Z} \mathrm{D}$.

In the first step of this expansion, we write $\alpha_{1}$ as a linear combination of the lex-first basis found in $\left\{D+\Phi_{T}(f): f \in \gamma\right\}$. The coefficients involved are the $v^{\sigma(1)} \cdot \alpha_{1}$ where $\sigma(1)$ varies over that lex-first basis. (We are beginning to build partial fractions schemata $\sigma$; so far we have specified the value at 1.) That gives an initial expansion of

$$
\begin{aligned}
\frac{\prod_{i=1}^{k} \alpha_{i}}{\prod_{f \in \gamma}\left(D+\Phi_{\mathrm{T}}(f)\right)} & =\left(\prod_{i=2}^{k} \alpha_{i}\right) \frac{\alpha_{1}}{\prod_{f \in \gamma}\left(D+\Phi_{\mathrm{T}}(f)\right)} \\
& =\left(\prod_{i=2}^{k} \alpha_{i}\right) \sum_{\sigma(1)} \frac{v^{\sigma(1)} \cdot \alpha_{1}}{\prod_{f \in \gamma \backslash\left\{f_{\sigma(1)}\right\}}\left(D+\Phi_{\mathrm{T}}(f)\right)}
\end{aligned}
$$

At this point we must split into cases, because the lex-first basis in $\left(D+\Phi_{\top}\left(f_{i}\right)\right.$ : $i=0, \ldots, \operatorname{dim} X, i \neq \sigma(1))$ depends on $\sigma(1)$.

Each time we bring in an $\alpha_{i}$, we linearly expand it in the lex-first basis in the remaining terms in the denominator. If there is no such basis, then as explained above the term may be dropped. Partial fractions expansion then eats each term from this basis in turn, and the choice of which one is recorded as $\sigma(i)$; the coefficient incurred is $v^{\sigma(i)} \cdot \alpha_{i}$. After doing this $k$ times, the final coefficient on $1 / \prod_{f \in \gamma^{\prime}}\left(D+\Phi_{T}(f)\right)$ is a sum over partial fraction schemata $\sigma$, of the product of $v^{\sigma(i)} \cdot \alpha_{i}$ :

$$
\begin{aligned}
\frac{\prod_{i=1}^{k} \alpha_{i}}{\prod_{f \in \gamma}\left(D+\Phi_{T}(f)\right)} & =\sum_{\sigma} \frac{\left(v^{\sigma(1)} \otimes \cdots \otimes v^{\sigma(k)}\right) \cdot\left(\alpha_{1} \otimes \cdots \otimes \alpha_{k}\right)}{\prod_{f \in \gamma \backslash\left\{f_{\sigma(i)}\right\}}\left(D+\Phi_{T}(f)\right)} \\
& =\sum_{\gamma^{\prime} \subseteq \gamma} \frac{\tau_{\gamma^{\prime}, \gamma} \cdot\left(\alpha_{1} \otimes \cdots \otimes \alpha_{k}\right)}{\prod_{f \in \gamma}\left(D+\Phi_{T}(f)\right)}
\end{aligned}
$$

up to terms dropped because, as explained above, they don't affect the measure near $p$.

We now sum over $\gamma$, then Fourier transform as in the proof above of theorem 1 , and we arrive at the complicated statement of theorem 3 . 


\section{Constraints on the COefficients $v_{\gamma}$}

4.1. An easy case of the multiplicities $v(Z)_{\left(f_{0}, f_{1}\right), \gamma}$. There is an important special case in which these multiplicities from theorem 2 are easy to compute.

Proposition 4.1. Let $\left\{v(Z)_{\left.\left(f_{0}, f_{1}\right), Y\right\}}\right.$ be as in theorem 2. By corollary 2.1, there exists an $\mathrm{S}$-equivariant map $\beta: \mathbb{P}^{1} \rightarrow \mathrm{X}$ such that $\beta(\infty)=\mathrm{f}_{1} \neq \beta(0)$.

Assume $\mathrm{Z}$ smooth at $\mathrm{f}_{1}$. Then the image of $\beta$ is $\overline{\mathrm{Z}^{\mathrm{f}_{1}}}$, a rational curve smooth away from $\beta(0)$.

Assume further that $\beta(0)=\mathrm{f}_{0}$. Then

$$
v(Z)_{\left(f_{0}, f_{1}\right), Y}=\operatorname{deg} \beta\left(\mathbb{P}^{1}\right)=\frac{\Phi_{S}\left(f_{1}\right)-\Phi_{S}\left(f_{0}\right)}{\left|S t a b_{S}\left(Z^{f_{1}}\right)\right|}=\frac{\Phi_{T}\left(f_{1}\right)-\Phi_{T}\left(f_{0}\right)}{-w t\left(T_{f_{1}} Z^{f_{1}}\right)}
$$

where $\operatorname{Stab}_{S}\left(Z^{f_{1}}\right)$ denotes the generic stabilizer subgroup scheme of $\mathrm{S}$ acting on $Z^{f_{1}}$, and $w t\left(T_{f_{1}} Z^{f_{1}}\right)$ denotes the $T$-weight on the tangent line $T_{f_{1}} Z^{f_{1}}$. (The numerator is a multiple thereof.)

Proof. Since $Z$ is smooth at $f_{1}$, so are $Z_{f_{1}}, Z^{f_{1}}$, with $\operatorname{dim} Z_{f_{1}}+\operatorname{dim} Z^{f_{1}}=\operatorname{dim} C$ where $C$ is the component of $Z$ containing $f_{1}$, and the intersection $Z_{f_{1}} \cap Z^{f_{1}}=\left\{f_{1}\right\}$ is transverse [BB76].

Since $Z_{f_{1}}$ is smooth and connected, its closure is irreducible, and $Y$ is supposed to be an irreducible component thereof. Hence $Y=\overline{Z_{f_{1}}}$ and is smooth at $f_{1}$. Similarly $\overline{Z^{f_{1}}}$ is irreducible and smooth at $f_{1}$. Since $Y$ is assumed to be codimension 1 in $Z$, we infer $\operatorname{dim} \overline{Z^{f_{1}}}=1$; since $\overline{Z^{f_{1}}}$ contains the curve $\beta\left(\mathbb{P}^{1}\right)$ they must be equal.

Let $b=0$ be the equation of a T-invariant hyperplane missing $f_{0}$ (existence guaranteed by lemma 2.4). We are attempting to determine the order of vanishing of $b$ along $Y=\overline{Z^{f_{1}}}$. By the transversality of the intersection $\overline{Z_{f_{1}}} \cap \overline{Z^{f_{1}}}$, we may instead restrict $b$ to $\beta\left(\mathbb{P}^{1}\right)$, and determine the order of vanishing of $b$ at the point $f_{1} \in \beta\left(\mathbb{P}^{1}\right)$.

This is the degree of the curve $\beta\left(\mathbb{P}^{1}\right)$, computed in terms of $\Phi_{S}$ in corollary 2.1. To compute in terms of $\Phi_{\top}$ requires that one extend the $S$-weight analysis in lemma 2.1 to the T-weights, which is straightforward.

The " $\beta(0)=f_{0}$ " condition in the proposition holds for flag manifolds (as follows from the next lemma), but is is not otherwise automatic. If $Z=F_{1}$ is the 
example from section 1.2 .2 , and $Y=\overline{Z_{b}}$, then $\overline{Z^{f_{1}}}$ is the $\mathbb{P}^{1}$ connecting $b$ and $c$; it doesn't make it down to d.

Corollary 4.1. Let $\mathrm{X}$ be smooth (and equidimensional), and assume each intersection $\mathrm{X}_{\mathrm{f}}^{\mathrm{g}}:=\mathrm{X}_{\mathrm{f}} \cap \mathrm{X}^{\mathrm{g}}$ is transverse. Then $\Delta(\mathrm{X}, \mathrm{S})$ is the order complex of the $\operatorname{poset}\left(X^{\top}, \geq\right)$.

Fix a maximal $\gamma$, and for each $i=1, \ldots, \operatorname{dim} \mathrm{X}$, assume that $\overline{\mathrm{X}_{\mathrm{f}_{\mathrm{i}-1}}}$ is smooth at $\mathrm{f}_{\mathrm{i}}$. Then each $\overline{\mathrm{X}_{\mathrm{f}_{\mathrm{i}-1}}^{\mathrm{f}_{\mathrm{i}}}}$ is a (possibly cuspidal) rational curve, and $v_{\gamma}=\prod_{i=1}^{\operatorname{dim} \mathrm{X}}$ $\operatorname{deg} \overline{X_{f_{i-1}}^{f_{i}}}$.

Proof. In [BB76] it is proven that this transversality condition implies that the B-B decomposition is a stratification. Hence $\overline{X_{f_{0}, \ldots, f_{k}}}=\overline{X_{f_{k}}}$ as long as $\left(f_{0}, \ldots, f_{k}\right)$ is a chain in $\left(X^{\top}, \geq\right)$, so nonempty for each chain. Therefore $\Delta(X, S)$ is the order complex.

We now show that under the S-equivariant map $\beta: \mathbb{P}^{1} \rightarrow \overline{X_{f_{i-1}}^{f_{i}}}$ constructed in proposition 4.1 , we have $\beta(0)=f_{i}$. For otherwise, $\overline{X_{f_{i-1}}} \supsetneq \overline{X_{\beta(0)}} \supsetneq \overline{X_{f_{i}}}$, with $\operatorname{dim} \overline{X_{f_{i}}} \leq \operatorname{dim} \overline{X_{f_{i-1}}}-2$ by lemma 3.4. But by lemma 3.7, $\operatorname{dim} X_{f_{i}}=\operatorname{dim} X_{f_{i-1}}-1$, contradiction.

The rest is proposition 4.1 and the recurrence in theorem 2 .

This extra smoothness, of $\overline{X_{f}}$ at each $g$ covering $f$, is known to hold for Schubert varieties (essentially from their normality). However, this corollary was proven in the symplectic situation [Kn99, theorem 1] without explicitly requiring this extra smoothness, so perhaps it is automatic.

We describe this story (from [Kn99]) in the case that $X$ is a flag manifold, though to recapitulate it properly would involve introducing a great deal of wholly standard notation, which we omit. When $Y \subset Z$ are Schubert varieties $\overline{X_{w r_{\beta}}} \subset$ $\overline{X_{w}} \subseteq \mathrm{G} / \mathrm{P}$ projectively embedded in the G-representation $V_{\lambda}$, the coefficient is $v(Z)_{\left(f_{0}, f_{1}\right), Y}=\left(w r_{\beta} \cdot \lambda-w \cdot \lambda\right) / \beta$. This is easily derived from the Chevalley-Monk rule for intersecting a Schubert variety with a hyperplane, and is the basic step in $[\mathrm{PS}]$.

4.2. Linear relations among the $\left\{v_{\gamma}\right\}$. The Duistermaat-Heckman function is piecewise polynomial, as can be seen from either their formula or theorem 1. However, theorem 1 hugely overestimates the number of pieces - it predicts 
a great many walls between regions of different polynomials that turn out to not actually be different. For example, in the case of a toric variety, the D-H function is 1 on the entire polytope, but theorem 1 breaks the polytope into a triangulation.

So anywhere within $\Phi_{\mathrm{T}}(\mathrm{X})$ that we know for some other reason there is not a jump in the D-H function - and we shall look nearby $\Phi_{\mathrm{T}}(\min (\mathrm{X}))$ - we get a linear condition among the coefficients $\left\{v_{\gamma}\right\}$. While the connection may be obscured by the Fourier transform, the proposition following is essentially built on this idea.

Proposition 4.2. Assume $\mathrm{X}$ has a unique supporting fixed point $\min (\mathrm{X})$. Let $\mathrm{C}_{\min (\mathrm{X})} \mathrm{X} \subseteq \mathrm{T}_{\min (\mathrm{X})} \mathrm{X}$ denote the tangent cone to $\mathrm{X}$ at $\min (\mathrm{X})$, and the tangent space to $\mathrm{X}$ at $\min (\mathrm{X})$, respectively. The ( $\mathrm{T}$-invariant) tangent cone carries a Chow class $\left[\mathrm{C}_{\min (\mathrm{X})} \mathrm{X}\right] \in \mathrm{A}_{\mathrm{T}}^{*}\left(\mathrm{~T}_{\min (\mathrm{X})} \mathrm{X}\right) \cong \operatorname{Sym}\left(\mathrm{T}^{*}\right)$. Denote by $\left[\{\overrightarrow{0}\} \in \mathrm{T}_{\min (\mathrm{X})} \mathrm{X}\right] \in$

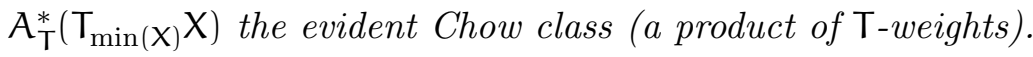

Then

$$
\begin{aligned}
& \sum_{\gamma} \frac{v_{\gamma}}{\prod_{\mathrm{f} \in \gamma, \mathrm{f} \neq \min (\mathrm{X})}\left(\Phi_{\mathrm{T}}(\mathrm{f})-\right.}\left.\Phi_{\mathrm{T}}(\min (\mathrm{X}))\right) \\
&=\frac{\left[\mathrm{C}_{\min (\mathrm{X})} \mathrm{X} \subseteq \mathrm{T}_{\min (\mathrm{X})} \mathrm{X}\right]}{\left[\{\overrightarrow{0}\} \in \mathrm{T}_{\min (\mathrm{X})} \mathrm{X}\right]} \text { as ratios in } \operatorname{Sym}\left(\mathrm{T}^{*}\right)
\end{aligned}
$$

where the sum is over maximum-length closure chains in $\mathrm{X}$.

Proof. By shrinking $\mathrm{V}$ to the linear span of $\widehat{X}$ and invoking lemma 2.3, we may assume that the $\Phi_{\mathrm{T}}(\min (\mathrm{X}))$-weight space in $\mathrm{V}$ is 1 -dimensional. That lets us invoke proposition 2.3 , which says that

$$
[\widehat{\mathrm{X}}] /[0 \times \mathrm{L}] \equiv\left[\mathrm{C}_{\min (\mathrm{X})} \mathrm{X} \subseteq \mathrm{T}_{\min (\mathrm{X})} \mathbb{P V}\right] /\left[\{\overrightarrow{0}\} \in \mathrm{T}_{\min (\mathrm{X})} \mathbb{P V}\right]
$$

as rational functions in $\operatorname{Sym}\left(T^{*}\right)[D]$ specialized at $D=-\Phi_{T}(f)$.

Theorem 4 gives us a formula for the left side of this equation, which specializes at $\mathrm{D}=-\Phi_{\mathrm{T}}(\mathrm{f})$ to the left side of the desired equation.

The right side is the equivariant multiplicity of $X$ at $\min (X)$, which can be computed inside either $\mathrm{T}_{\min (\mathrm{X})} \mathbb{P} \mathrm{V}$ or $\mathrm{T}_{\min (\mathrm{X})} \mathrm{X}$.

Corollary 4.2. Assume in addition that there exists a set $\mathrm{Q} \subseteq \mathrm{X}^{\top}$ of fixed points such that the weights in $\mathrm{T}_{\min (\mathrm{X})} \mathrm{X}$, with repetition, are $\left\{\Phi_{\mathrm{T}}(\mathbf{q})-\Phi_{\mathrm{T}}(\min (\mathrm{X})): \mathrm{q} \in\right.$ 
$\mathrm{Q}$. Then this formula can be rewritten inside $\operatorname{Sym}\left(\mathrm{T}^{*}\right)$ as

$$
\begin{aligned}
\sum_{\gamma} v_{\gamma} \prod_{f \in X^{\top} \backslash \gamma}\left(\Phi_{\mathrm{T}}(f)-\Phi_{\mathrm{T}}(\min (\mathrm{X}))\right) \\
=\left[\mathrm{C}_{\min (\mathrm{X})} \mathrm{X} \subseteq \mathrm{T}_{\min (\mathrm{X})} \mathrm{X}\right] \prod_{\substack{\mathrm{f} \in \mathrm{X}^{\top} \\
\mathrm{f} \notin \mathrm{Q} \cup\{\min (\mathrm{X})\}}}\left(\Phi_{\mathrm{T}}(\mathrm{f})-\Phi_{\mathrm{T}}(\min (\mathrm{X}))\right) .
\end{aligned}
$$

Let $\check{\sigma}: \mathrm{T}^{*} \rightarrow \mathbb{Z}$ be a linear functional, and write $\Phi_{\mathrm{R}}:=\check{\sigma} \circ \Phi_{\mathrm{T}}$. Assume that $\delta:=\left\{f \in X^{\top}: \Phi_{R}(f)=\Phi_{R}(\min (X))\right\}$ is a closure chain, and that $\delta \nsubseteq Q \cup\{\min (X)\}$. Then

$$
\sum_{\gamma \supseteq \delta} v_{\gamma} \prod_{f \in X^{\top} \backslash \gamma}\left(\Phi_{R}(f)-\Phi_{R}(\min (X))\right)=0
$$

where the left side is a sum over maximum-length closure chains.

Proof. To get the first formula above, multiply both sides of the one from proposition 4.2 by $\prod_{\mathrm{f} \in \mathrm{X}^{\mathrm{T}}, \mathrm{f} \neq \min (\mathrm{X})}\left(\Phi_{\mathrm{T}}(\mathrm{f})-\Phi_{\mathrm{T}}(\min (\mathrm{X}))\right)$.

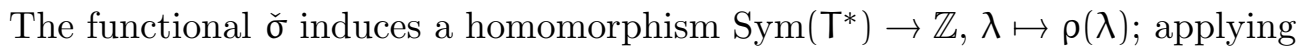
it to the first formula we get the equation

$$
\begin{aligned}
\sum_{\gamma} v_{\gamma} \prod_{f \in X^{\top} \backslash \gamma}\left(\Phi_{R}(f)-\Phi_{R}(\min (X))\right) & \\
=\left[C_{\min (X)} X \subseteq T_{\min (X)} X\right] & \prod_{\substack{f \in X^{\top} \\
f \notin Q \cup\{\min (X)\}}}\left(\Phi_{R}(f)-\Phi_{R}(\min (X))\right) .
\end{aligned}
$$

For any $\gamma \nsupseteq \delta$, one of the terms in the product $\prod_{f \in X^{\top} \backslash \gamma}\left(\Phi_{R}(f)-\Phi_{R}(\min (X))\right)$ is zero, so on the left side it is enough to sum over $\gamma \supseteq \delta$.

By the condition $\delta \nsubseteq \mathrm{Q} \cup\{\min (\mathrm{X})\}$, one of the terms in the right-hand product is zero.

Some remarks:

- We used the notation $\Phi_{R}$ because the Pontrjagin dual of $\check{o}$ is a homomorphism $R: \mathbb{G}_{m} \rightarrow T$, whose moment map on the fixed points is this $\Phi_{\mathrm{R}}$.

- One can obtain many more such conditions by applying corollary 4.2 to components of $\overline{X_{f_{0}, \ldots, f_{k}}}$ of codimension $k$ in $X$, and using theorem 2 . 
- If $X$ is irreducible, we can study instead its image under the $\beta$ from proposition 2.4 (picking up a factor from the degree of $\beta$ to its image). In this smaller projective space, it is easy to see that a set $\mathrm{Q}$ as postulated in corollary 4.2 must exist, even for $T_{\min (X)} \mathbb{P V} \geq T_{\min (X)} X$.

- If $X$ has not only isolated fixed points but isolated fixed curves, as in [GKM98], then this set $\mathrm{Q}$ exists canonically: take the T-fixed points other than $\min (X)$ on the T-fixed curves passing through $\min (X)$. This condition holds for flag manifolds and toric varieties, though not for the Bott-Samelson manifold from section 1.2.5.

As usual, things are particularly simple for $\mathrm{X}$ a toric variety, where the simplicial complex $\Delta(X, S)$ is a triangulation of the moment polytope $P$, whose vertices correspond naturally to $\mathrm{X}^{\top}$. The set $\mathrm{Q}$ can (and must) be taken to be the vertices sharing an edge with $\min (X)$.

4.3. Assembling the coefficients $\left\{v_{\bar{\gamma}}\right\}$. The formula $\operatorname{deg} X=\sum_{\gamma} v_{\gamma}$ mentioned after theorem 1, summing over maximum-length closure chains, can be refined to $\operatorname{deg} \mathrm{X}=\sum_{\bar{\gamma}} \nu_{\bar{\gamma}}$ summing over maximum-length witnesses. We now give an inductive version of this formula.

Given a closure chain $\gamma=\left(f_{0}, \ldots, f_{k}\right)$ and a component $Y$ of $\overline{X_{f_{0}, \ldots, f_{k}}}$ of dimension $\operatorname{dim} X-k$ (which requires $\gamma$ to be the initial segment of a maximum-length chain), define

$$
v_{\gamma, \mathrm{Y}}:=\sum_{\bar{\gamma}=\left(\mathrm{f}_{\mathrm{O}} \in \mathrm{Y}_{0}, \ldots, \mathrm{f}_{\mathrm{k}} \in \mathrm{Y}_{\mathrm{k}}=\mathrm{Y}\right)} v(\mathrm{X})_{\bar{\gamma}}
$$

where the summands were defined in section 3.2. If $k=\operatorname{dim} X$, then $Y=\left\{f_{\operatorname{dim} X}\right\}$ by lemma 3.7 , and therefore $v_{\gamma, Y}$ is the $v_{\gamma}$ also defined in section 3.2 .

Proposition 4.3. Fix $\gamma=\left(f_{0}, \ldots, f_{k}\right)$ and a component $Y$ of $\overline{X_{\mathrm{f}_{0}, \ldots, f_{k}}}$ of dimension $\operatorname{dim} \mathrm{X}-\mathrm{k}$. Pick a $\mathrm{T}$-invariant hyperplane $\mathbb{P H}$ not containing $\mathrm{f}_{\mathrm{k}}$, and let $\left\{\mathrm{Z}_{\mathrm{i}}\right\}$ be the irreducible components of $\mathrm{Y}_{\mathrm{k}} \cap \mathbb{P H}$. Then

$$
v_{\left(f_{0}, \ldots, f_{k}\right), Y} \operatorname{deg} Y=\sum_{i} v_{\left(f_{0}, \ldots, f_{k}, \min \left(Z_{i}\right)\right), Z_{i}} \operatorname{deg} Z_{i} .
$$

Proof.

$$
\operatorname{deg} Y_{k}=\operatorname{deg}\left(Y_{k} \cap \mathbb{P H}\right)=\sum_{i} m_{i} \operatorname{deg}\left(Z_{i}\right)
$$


where the $m_{i}$ are the multiplicities of the components $Z_{i}$ in the scheme $Y_{k} \cap \mathbb{P H}$. The result follows by unwinding the definition of $v(X)_{\bar{\gamma}}$.

\section{ACKNOWLEDGEMENTS}

This project has been gestating since shortly after [Kn99], and indeed this paper might be considered only a progress report toward $[\mathrm{Kn}]$. In that long period I have discussed it with many people. I best remember having useful discussions with Michel Brion (to whom I am particularly grateful for remarks on previous versions of this article), Rebecca Goldin, Francisco Santos, and Catalin Zara, and with Mark Haiman and Bernd Sturmfels about the Hilbert scheme of $n$ points in the plane.

\section{REFERENCES}

[Ak81] E. Akyıldız, Bruhat decomposition via $G_{m}$-action, Bull. Acad. Polon. Sci. Ser. Sci. Math. 28 (1980), no. 11-12, 541-547 (1981).

[AK] V. Alexeev, A. Knutson, Complete moduli spaces of branchvarieties, preprint 2006. math.AG/0602626

[AB84] M. Atiyah, R. Bott, The moment map and equivariant cohomology, Topology 23 (1984) no. 1, 1-28.

[BV84] N. Berline, M. Vergne, Un calcul de l'indice équivariant de l'opérateur de Dirac par la méthode de la chaleur, C. R. Acad. Sci. Paris Sér. I Math. 299 (1984), no. 11, 511-514.

[BB76] A. Białynicki-Birula, Some properties of the decompositions of algebraic varieties determined by actions of a torus, Bull. Acad. Polon. Sci. Ser. Sci. Math. Astronom. Phys. 24 (1976), no. 9, 667-674.

[BW82] A. Björner, M. Wachs, Bruhat order of Coxeter groups and shellability, Adv. in Math. 43 (1982), no. 1, 87-100.

[BP90] M. Brion, C. Procesi, Action d'un tore dans une variété projective, Operator algebras, Unitary Representations, Enveloping Algebras, and Invariant Theory, Progress in Mathematics Vol. 92, (1990) Birkhä user, pp. 509-539.

[Br97] M. Brion, Equivariant Chow groups for torus actions, Transformation Groups, Vol. 2, No. 3, 1997, pp. 225-267.

[Br98] _ Equivariant cohomology and equivariant intersection theory, Notes de l'école d'été "Théories des représentations et géométrie algébrique" (Montréal, 1997). math. AG/9802063

[DCP73] C. DeConcini, C. Procesi, Complete symmetric varieties. Lecture Notes in Math. 996, Springer, 1973, 1-44.

[Du03] H. Duan, The degree of a Schubert variety, Adv. Math. 180 (2003), no. 1, 112-133. 
[DH82] J. J. Duistermaat, G. Heckman, On the variation in the cohomology class of the symplectic form of the reduced phase space, Invent. Math. 69 (1982), no. 2, 259-268.

[GM06] R. Goldin, S. Martin, Cohomology pairings on the symplectic reduction of products, Canad. J. Math. 58 (2006), no. 2, 362-380. math.SG/0408250

[GKM98] M. Goresky, R. Kottwitz, R. MacPherson, Equivariant cohomology, Koszul duality, and the localization theorem, Invent. Math. 131 (1998), no. 1, 25-83.

[Gu94] V. Guillemin, Reduced phase spaces and Riemann-Roch, Lie theory and geometry, 305334, Progr. Math., 123, Birkhäuser Boston, Boston, MA, 1994.

[GLS88] _ E. Lerman, S. Sternberg, On the Kostant multiplicity formula, J. Geom. Phys. 5 (1988), no. 4, 721-750.

[GS95] _ S. Sternberg, The coefficients of the Duistermaat-Heckman polynomial, Geometry, Topology, and Physics for Raoul Bott, Conference proceedings and lecture notes in geometry and topology, Vol. IV, International Press, 1995, 202-213.

[Ha02] M. Haiman, Combinatorics, symmetric functions and Hilbert schemes, Current Developments in Mathematics 2002, no. 1 (2002), 39-111.

[He81] W. Hesselink, Concentration under actions of algebraic groups, Paul Dubreil and MariePaule Malliavin Algebra Seminar, 33rd Year (Paris, 1980), pp. 5589, Lecture Notes in Math., 867, Springer, Berlin, 1981.

[Jo97] A. Joseph, Orbital varieties, Goldie rank polynomials and unitary highest weight modules, Algebraic and analytic methods in representation theory (Sønderborg, 1994), 53-98.

[Ju77] J. Jurkiewicz, An example of algebraic torus action which determines the nonfiltrable decomposition, Bull. Acad. Polon. Sci. Sér. Sci. Math. Astronom. Phys. 25 (1977), no. 11, 1089-1092.

[Ki86] F. Kirwan, Cohomology of quotients in symplectic and algebraic geometry, Mathematical Notes 31, Princeton University Press, Princeton, NJ, 1984.

[Kn99] A. Knutson, A Littelmann-type formula for Duistermaat-Heckman measures, Inventiones mathematicae, 135 (1999) no. 1, 185-200.

[Kn06] _ Balanced normal cones and Fulton-MacPherson's intersection theory,

Pure and Applied Mathematics Quarterly Vol 2, \# 4, 2006 (MacPherson issue part II)

$[\mathrm{Kn}] \_$, Standard bases for homogeneous coordinate rings, in preparation.

$[\mathrm{KMY}] \ldots$, E. Miller and A. Yong, Gröbner geometry of vertex decompositions and of flagged tableaux, To appear in Crelle's journal, math.AG/0502144.

[KZJ07] _ P. Zinn-Justin, A scheme related to the Brauer loop model, Advances in Mathematics 214 (2007), Issue 1, 40-77.

[Ko78] J. Konarski, Decompositions of normal algebraic varieties determined by an action of a one-dimensional torus, Bull. Acad. Polon. Sci. Sér. Sci. Math. Astronom. Phys. 26 (1978), no. 4, 295-300.

[PS] A. Postnikov, R. Stanley, Chains in the Bruhat order, preprint. math.C0/0502363

[Ro89] W. Rossmann, Equivariant multiplicities on complex varieties, Orbites unipotentes et représentations, III, Astérisque no. 173-174, (1989), 11, 313-330. 
Allen Knutson

Department of Mathematics

Cornell University

Ithaca, NY14853-4201

USA

E-mail: allenk@math.cornell.edu 